\title{
PROPERTIES OF THE SEQUENCE OF CLOSED POWERS OF A MAXIMAL IDEAL IN A SUP-NORM ALGEBRA $\left({ }^{1}\right)$
}

\author{
BY \\ S. J. SIDNEY $\left({ }^{2}\right)$
}

1. A sup-norm algebra is a closed point-separating subalgebra $A$ of $C(X)$ containing the constants, where $X$ is a compact Hausdorff space. $M_{A}$ will denote the (compact Hausdorff) space of multiplicative linear functionals $\varphi$ on $A$ in the Gelfand topology, and to each such $\varphi$ we associate its kernel $A_{\varphi}$; the $A_{\varphi}$ are precisely the maximal ideals of $A$. $X$ can be viewed as a closed subset of $M_{A}$, and there is an isomorphism $f \rightarrow \hat{f}$ of $A$ into $C\left(M_{A}\right)$ such that $\hat{f} \mid X=f$. The isomorphism is given by $\hat{f}(\varphi)=\varphi(f)$. We will frequently write $f$ for $\hat{f}$. $S_{A}$ will denote the Silov boundary of $A$, that is, the smallest closed subset of $X$ on which each function in $A$ attains its maximum modulus. Each $f \in A$ has the same supremum over $S_{A}, X$, and $M_{A}$; this common supremum is the norm of $f$, written $\|f\|$.

We intend to study the ideals $\left(A_{\varphi}^{n}\right)^{-}$. [Note: If $I$ is an ideal in $A, I^{n}$ is the ideal generated by products $f_{1} \cdots f_{n}$ with $f_{i} \in I$ if $n \geqq 1$, and $I^{0}=A$; also $\bar{I}$ denotes the closure of $I$.] Evidently $\left(A_{\varphi}^{n}\right)^{-} \supset\left(A_{\varphi}^{n+1}\right)^{-}$and if the inclusion is an equality for some $n$, then it is an equality for all subsequent $n$. A problem of the sort that will interest us-indeed, in great part the stimulus for this research-is to determine whether one can preassign the cutoff point in this chain of inclusions: given $n$, is it possible to have $\left(A_{\varphi}^{n}\right)^{-} \neq\left(A_{\varphi}^{n+1}\right)^{-}$but $\left(A_{\varphi}^{n+1}\right)^{-}=\left(A_{\varphi}^{n+2}\right)^{-}$?

More generally, how much control do we have over the sequence of numbers $\left\{\operatorname{dim}\left(\left(A_{\varphi}^{n}\right)^{-} /\left(A_{\varphi}^{n+1}\right)^{-}\right): n=1,2, \ldots\right\}$ ? While we will not give an explicit arithmetic answer, we will reduce the problem to a purely algebraic one. In particular, it is possible to obtain any nonincreasing sequence of nonnegative integers, and hence to preassign the cutoff point.

We now will begin to formulate the problem we will actually solve. First, consider a graded algebra (all algebras in this paper are commutative algebras over the complex field $C$ ) $Q=\sum_{n=0}^{\infty} \oplus Q_{n}$ and suppose that $Q_{0}$ consists of scalar multiples of an identity for $Q$, and that $Q_{0}$ and $Q_{1}$ generate $Q$. If $\left\{F_{\gamma}: \gamma \in \Gamma\right\}$ is a basis for $Q_{1}$ and $R=C\left[\left\{X_{\gamma}: \gamma \in \Gamma\right\}\right]$ is the polynomial ring in $\Gamma$ variables with its usual graded algebra structure, the linear extension of the map $X_{\gamma_{1}} \cdots X_{\gamma_{n}} \rightarrow F_{\gamma_{1}} \cdots F_{\gamma_{n}}$ is a graded algebra homomorphism of $R$ onto $Q$. Thus $Q$ is isomorphic as a

Received by the editors December 20, 1966.

(1) This work comprises a portion of the author's doctoral dissertation [S2], written under the direction of Professors Andrew M. Gleason and Lynn H. Loomis and accepted by Harvard University in June 1966.

$\left({ }^{2}\right)$ Research partially supported by National Science Foundation Graduate Fellowship. 
graded algebra to $R / J$ where $J$ is some homogeneous ideal in $R$. (For algebraic terminology and theorems consult [ZS].)

Next, suppose $B$ is a linear algebra and let $\left\{I_{n}: n=0,1,2, \ldots\right\}$ be a sequence of ideals in $B$ such that $I_{0}=B, I_{n+1} \subset I_{n}$, and $I_{p} I_{q} \subset I_{p+q}$. We associate with $\left\{I_{n}\right\}$ a graded vector space $V=\sum_{n=0}^{\infty} \oplus V_{n}$ where $l_{n}: I_{n} \rightarrow V_{n}$ is some fixed linear isomorphism of $I_{n}$ onto a space $V_{n}$. Let $U=\sum_{n=0}^{\infty} \oplus U_{n}$ where $U_{n}=l_{n}\left(I_{n+1}\right) \subset V_{n}$, and let $Q$ be the quotient graded vector space $Q=V / U=\sum_{n=0}^{\infty} \oplus Q_{n}$ where $Q_{n}=V_{n} / U_{n}$. $V$ is a graded algebra if we extend linearly the map given by $b * c=l_{p+q}\left(l_{p}^{-1}(b) \cdot l_{q}^{-1}(c)\right)$ for $b \in V_{p}$ and $c \in V_{q}$, and $U$ is a homogeneous ideal of $V$; hence the multiplication $\left(b+U_{p}\right) *\left(c+U_{q}\right)=b * c+U_{p+q}$ for $b \in V_{p}$ and $c \in V_{q}$ makes $Q$ into a graded algebra.

Let us now apply this chain of ideas to the situation when $B$ is a sup-norm algebra $A$ and $I_{n}=\left(A_{\varphi}^{n}\right)^{-}$for some $\varphi \in M_{A}$. Associate to this situation a graded algebra $Q$ as in the last paragraph, so $Q_{n}=\left(A_{\varphi}^{n}\right)^{-} /\left(A_{\varphi}^{n+1}\right)^{-}$. Evidently $Q_{0}$ consists of scalar multiples of an identity for $Q$. In general it is false that $Q_{0}$ and $Q_{1}$ generate $Q$. (The closure of the polynomials on a countable product of discs yields a counterexample.) However, if $Q_{1}$ has finite dimension, say $r$, we can show that $Q_{0}$ and $Q_{1}$ generate $Q$. This amounts to showing that for $n>1$ the products $b_{1} * b_{2} * \cdots * b_{n}$ with $b_{i} \subset Q_{1}$ span $Q_{n}$. Let $Q_{n}^{\prime}$ denote the span of these products. $Q_{n}^{\prime}$ is a finitedimensional subspace of $Q_{n}$, hence is closed in $Q_{n}$ where the latter has its norm topology as $\left(A_{\varphi}^{n}\right)^{-} /\left(A_{\varphi}^{n+1}\right)^{-}$. It remains to prove density of $Q_{n}^{\prime}$ in $Q_{n}$.

Let $F_{1}, \ldots, F_{r}$ be a basis for $Q_{1}$ and let $F_{j}$ have the representative $f_{j} \in A_{\varphi}$. Let $f \in\left(A_{\varphi}^{n}\right)^{-}$. Select a sequence $\left\{g_{k}\right\}$ in $A_{\varphi}^{n}$ such that $\left\|f-g_{k}\right\| \rightarrow 0$. Write $g_{k}=\sum_{i=1}^{t_{k}} g_{k i 1}$ $\cdots g_{k i n}$ where $g_{k i j} \in A_{\varphi}$. Write $g_{k i j}=\sum_{s=1}^{r} \alpha_{k i j s} f_{s}+h_{k i j}$ where $h_{k i j} \in\left(A_{\varphi}^{2}\right)^{-}$. Then

$$
l_{n}\left(g_{k i 1} \cdots g_{k i n}\right)+U_{n}=l_{n}\left(\prod_{j=1}^{n}\left(\sum_{s=1}^{r} \alpha_{k i j s} f_{s}\right)\right)+U_{n}=\prod_{j=1}^{n}\left(\sum_{s=1}^{r} \alpha_{k i j s} F_{s}\right) \in Q_{n}^{\prime}
$$

so $l_{n}\left(g_{k}\right)+U_{n} \in Q_{n}^{\prime}$. But $\left\|\left(l_{n}(f)+U_{n}\right)-\left(l_{n}\left(g_{k}\right)+U_{n}\right)\right\| \leqq\left\|f-g_{k}\right\| \rightarrow 0$, so $l_{n}(f)+U_{n}$ is in the closure of $Q_{n}^{\prime}$. Thus $Q_{n}^{\prime}$ is dense in $Q_{n}$, hence $Q_{n}^{\prime}=Q_{n}$. Therefore $Q_{0}$ and $Q_{1}$ generate $Q$.

Combining with our first remarks about graded algebras, we see that we have proved the following:

TheOREM 1. Let $A$ be a sup-norm algebra, $\varphi \in M_{A}$. Then the direct sum $\sum_{n=0}^{\infty} \oplus\left(\left(A_{\varphi}^{n}\right)^{-} /\left(A_{\varphi}^{n+1}\right)^{-}\right)$has in a natural way the structure of a graded algebra $Q$. If $\operatorname{dim}\left(A_{\varphi} /\left(A_{\varphi}^{2}\right)^{-}\right)=r<\infty$ then $Q$ is isomorphic as a graded algebra to $C\left[X_{1}, \ldots, X_{r}\right] / J$ where $J$ is a homogeneous ideal in $C\left[X_{1}, \ldots, X_{r}\right]$ and $J_{0}=J_{1}=\{0\}$.

Note that we really only require $A$ to be a Banach algebra with identity. Further, the same arguments work if $A$ is simply a linear algebra with identity, $A_{\varphi}$ is the kernel of a multiplicative linear functional $\varphi$, and we take $I_{n}=\left(A_{\varphi}\right)^{n}$ (rather than $\left.\left(A_{\varphi}^{n}\right)^{-}\right)$; and in this case no finiteness hypothesis is required. 
A consequence of Theorem 1 is our first quantitative information: if

$$
\operatorname{dim}\left(A_{\varphi} /\left(A_{\varphi}^{2}\right)^{-}\right)=r<\infty
$$

then $\operatorname{dim}\left(\left(A_{\varphi}^{n}\right)^{-} /\left(A_{\varphi}^{n+1}\right)^{-}\right) \leqq C_{n+r-1, n}$ for all $n$. In addition, the Hilbert-Serre theorem [ZS, p. 232] says that there is a polynomial $G$ of degree at most $r-1$ such that $\operatorname{dim}\left(\left(A_{\varphi}^{n}\right)^{-} /\left(A_{\varphi}^{n+1}\right)^{-}\right)=G(n)$ for $n$ large. (By convention, the zero polynomial has degree -1 .)

Theorem 1 can be radically refined if $\varphi$ has a unique (nonnegative regular Borel) representing measure on $X$ (e.g., if $A$ is Dirichlet or, more generally, logmodular). It is then known [S1] that either $Q$ is 1-dimensional (i.e., $Q_{n}=0$ for $n>0$ ) or $Q$ is isomorphic to $C\left[X_{1}\right]$, according as $\varphi$ is or is not a one-point part for $A$.

The main objective of this paper is to obtain a converse to Theorem $1:$ if $J$ is a homogeneous ideal in $C\left[X_{1}, \ldots, X_{r}\right]$, we find a sup-norm algebra $A$ and $\varphi \in M_{A}$ whose corresponding graded algebra is isomorphic to $C\left[X_{1}, \ldots, X_{r}\right] / J$. $\S \S 2$ and 3 are devoted to this, and it is accomplished in Theorem 3.17. In $\S 4$ we show that the algebras constructed to prove Theorem 3.17 are actually local algebras.

The construction we use, which is developed in $\$ 3$, is useful for producing examples of many pathologies concerned with the $\left(A_{\varphi}^{n}\right)^{-}$. For example, one can construct a one-point part $\varphi$ supporting a nontrivial bounded derivation (Example 5.13 below). $\S 5$ is devoted to exploration of the types of algebra to which our construction leads, and to examples.

Let me record here my debt to Professors E. L. Stout and Lynn H. Loomis for valuable consultation during this research; and most especially to Professor Andrew M. Gleason, who gave this work its initial stimulus both by suggesting the study of derivations and by showing the author the algebra (Example 5.14 below) which motivated our construction, and whose ideas have played an important role throughout.

2. We will have to know something about tensor products, and this section is devoted to the relevant material. For our purposes it consists entirely of technical lemmas. The early material is standard, and proofs are omitted; the reader ought to be able to fill them in, or he can consult the literature (see for example [Ge]). The later material on the interplay between tensor products and direct sums is probably new, but is quite easy.

Let $\Gamma$ be an index set, and for each $\gamma \in \Gamma$, let $X_{\gamma}$ be a compact Hausdorff space. If for each $\gamma$ we are given $f_{\gamma} \in C\left(X_{\gamma}\right)$ such that $f_{\gamma}=1$ for all but finitely many $\gamma$, define a function $\otimes\left\{f_{\gamma}\right\}$ on $X=\prod X_{\gamma}$ by

$$
\left(\otimes\left\{f_{\gamma}\right\}\right)\left(\left\{x_{\gamma}\right\}\right)=\prod f_{\gamma}\left(x_{\gamma}\right) \text {. }
$$

$X$ is a compact Hausdorff space in the product topology, and $\otimes\left\{f_{\gamma}\right\} \in C(X)$.

If $W$ is a subset of a vector space $V, \operatorname{sp}(W)$ will denote the linear span of $W$; if $V$ is also a topological vector space, $\mathrm{sp}(W)^{-}$is the closure of $\mathrm{sp}(W)$. Suppose for each $\gamma$ we are given a nonempty subset $W_{y}$ of $C\left(X_{\gamma}\right)$, and suppose $W_{\gamma}$ contains 
1 for all but finitely many $\gamma \cdot \otimes\left\{W_{\gamma}\right\}=\operatorname{sp}\left\{\otimes\left\{f_{\gamma}\right\}: f_{\gamma} \in W_{\gamma}\right\}$ is the tensor product of the $W_{\gamma}$ and $\otimes\left\{W_{\gamma}\right\}^{-}$is its closure, the closed tensor product of the $W_{\gamma}$. Clearly $\bigotimes\left\{W_{\gamma}\right\}=\otimes\left\{\operatorname{sp}\left(W_{\gamma}\right)\right\}$ and $\otimes\left\{W_{\gamma}\right\}^{-}=\otimes\left\{W_{\gamma}^{-}\right\}^{-}$.

Should $\Gamma$ be finite we can write $X=X_{1} \times \cdots \times X_{n}, f_{1} \otimes \cdots \otimes f_{n}$ and $W_{1} \otimes \cdots$ $\otimes W_{n}$ with the obvious meanings.

If $\Gamma$ is a disjoint union $\Gamma=\bigcup\left\{\Gamma_{\sigma}: \sigma \in \Sigma\right\}$ where $\Gamma_{\sigma}$ is nonempty, there are obvious natural identifications

$$
\bigotimes\left\{W_{\gamma}: \gamma \in \Gamma\right\}=\bigotimes\left\{\bigotimes\left\{W_{\gamma}: \gamma \in \Gamma_{\sigma}\right\}: \sigma \in \Sigma\right\}
$$

and

$$
\begin{aligned}
\bigotimes\left\{W_{\gamma}: \gamma \in \Gamma\right\}^{-} & =\bigotimes\left\{\bigotimes\left\{W_{\gamma}: \gamma \in \Gamma_{\sigma}\right\}: \sigma \in \Sigma\right\}^{-} \\
& =\bigotimes\left\{\bigotimes\left\{W_{\gamma}: \gamma \in \Gamma_{\sigma}\right\}^{-}: \sigma \in \Sigma\right\}^{-} .
\end{aligned}
$$

Let $\Gamma$ be a disjoint union $\Gamma=\Gamma^{1} \cup \Gamma^{2}$ with $\Gamma^{j}$ nonempty. Write

$$
X^{j}=\prod\left\{X_{\gamma}: \gamma \in \Gamma^{j}\right\}
$$

so $X=X^{1} \times X^{2}$ in a natural fashion. For each $x^{2} \in X^{2}$ we get an imbedding $e_{x^{2}}: X^{1} \rightarrow X$ by $e_{x^{2}}\left(x^{1}\right)=\left(x^{1}, x^{2}\right)$, and the $e_{x^{2}}\left(X^{1}\right)$ for various $x^{2}$ provide a disjoint covering of $X$. The dual map $e_{x}^{*_{2}}: C(X) \rightarrow C\left(X^{1}\right)$ is a norm-decreasing algebra homomorphism.

LEMMA 2.1. $e_{x^{2}}^{*}\left(\bigotimes\left\{W_{\gamma}: \gamma \in \Gamma\right\}\right) \subset \bigotimes\left\{W_{y}: \gamma \in \Gamma^{1}\right\}$ and $e_{x^{2}}^{*}\left(\otimes\left\{W_{y}: \gamma \in \Gamma\right\}^{-}\right)$ $\subset \bigotimes\left\{W_{\gamma}: \gamma \in \Gamma^{1}\right\}^{-}$. If there exists for each $\gamma \in \Gamma^{2}$ an $f_{\gamma} \in W_{\gamma}$ such that $f_{\gamma}\left(x_{\gamma}^{2}\right) \neq 0$, then these inclusions are actually equalities.

In particular, if $\gamma_{0} \in \Gamma$ then as a function of $x_{\gamma_{0}}$ in $X_{\gamma_{0}}$ alone, each $f \in \bigotimes\left\{W_{\gamma}\right\}$ is in $\operatorname{sp}\left(W_{\gamma_{0}}\right)$ and each $f \in \bigotimes\left\{W_{\gamma}\right\}^{-}$is in $\operatorname{sp}\left(W_{\gamma_{0}}\right)^{-}$.

For each $\gamma$ let $Y_{\gamma}$ be a nonempty closed subset of $X_{\gamma}$ and let $Y=\prod Y_{\gamma}$. The inclusions $l_{\gamma}: Y_{\gamma} \rightarrow X_{\gamma}$ induce an inclusion $l: Y \rightarrow X$ and the dual map $l^{*}: C(X)$ $\rightarrow C(Y)$ where $l^{*}(f)=f \mid Y$.

LEMMA 2.2. $l^{*}$ is a norm-decreasing algebra homomorphism, $l^{*}\left(\otimes\left\{W_{\gamma}\right\}\right)$ $=\bigotimes\left\{W_{\gamma} \mid Y_{\gamma}\right\}$, and $l^{*}\left(\bigotimes\left\{W_{\gamma}\right\}^{-}\right) \subset \otimes\left\{W_{\gamma} \mid Y_{\gamma}\right\}^{-}$. If $\gamma \in \Gamma$ and $f \in \operatorname{sp}\left(W_{\gamma}\right)$ imply $\|f\|_{Y_{y}}=\|f\|_{X_{y}}$ then $l^{*} \mid\left(\otimes\left\{W_{y}\right\}^{-}\right)$is norm-preserving and $l^{*}\left(\bigotimes\left\{W_{y}\right\}^{-}\right)=\bigotimes\left\{W_{y} \mid Y_{y}\right\}^{-}$. (The norms are the obvious suprema.)

If $W_{\gamma}=A_{\gamma}$ a sup-norm algebra on $X_{\gamma}$, then $\otimes\left\{A_{\gamma}\right\}$ is a point-separating algebra with 1 on $\prod X_{y}$ and $\otimes\left\{A_{\gamma}\right\}^{-}$is a sup-norm algebra on $\prod X_{\gamma} . S_{A_{y}} \subset X_{\gamma} \subset M_{A_{y}}$ and we can think of $A_{\gamma}$ as a sup-norm algebra on $M_{A_{\gamma}}$. Let $l_{1}: \Pi S_{A_{y}} \rightarrow \Pi X_{\gamma}$ and $l_{2}: \prod X_{\gamma} \rightarrow \prod M_{A_{y}}$ be the inclusions, with dual maps $l_{1}^{*}: C\left(\prod X_{\gamma}\right) \rightarrow C\left(\prod S_{A_{y}}\right)$ and $l_{2}^{*}: C\left(\prod M_{A_{y}}\right) \rightarrow C\left(\prod X_{\gamma}\right)$. Lemma 2.2 leads to

THEOREM 2.3. $l_{1}^{*}$ maps $\otimes\left\{A_{\gamma} \mid X_{\gamma}\right\}^{-}$isometrically and isomorphically onto $\otimes\left\{A_{\gamma} \mid S_{A_{y}}\right\}^{-} . l_{2}^{*}$ maps $\otimes\left\{A_{\gamma}\right\}^{-}$isometrically and isomorphically onto $\otimes\left\{A_{y} \mid X_{\gamma}\right\}^{-}$. $M_{\otimes\left\{A_{\gamma}\right\}^{-}}=\prod M_{A_{\gamma}}$ and $S_{\otimes\left\{A_{\gamma}\right\}^{-}}=\prod S_{A_{\gamma}}$. 
Our emphasis will now shift to the behavior of subspaces in tensor products. The set $\Gamma$ will, until notice to the contrary is given, have cardinality 2 .

Let $V_{1}, \ldots, V_{n}$ be subspaces of a vector space $V$. In addition to their vector space sum $\operatorname{sp}\left(V_{1} \cup \cdots \cup V_{n}\right)=V_{1}+\cdots+V_{n} \subset V$, we can define their direct sum $V_{1} \oplus \cdots \oplus V_{n}$. This, of course, is not a subspace of $V$; it is the space of $n$-tuples $\left(v_{1}, \ldots, v_{n}\right)$ where $v_{j} \in V_{j}$ with coordinate-wise addition and scalar multiplication. There is an obvious natural map of $V_{1} \oplus \cdots \oplus V_{n}$ into $V$, and its image is $V_{1}+\cdots+V_{n}$. If this map is injective, we write $V_{1} \oplus \cdots \oplus V_{n} \equiv V_{1}+\cdots+V_{n}$. If $V_{0}$ is another subspace of $V$, the expression $V_{1} \oplus \cdots \oplus V_{n} \equiv V_{0}$ means $V_{0}=V_{1}+\cdots$ $+V_{n}$ and $V_{1} \oplus \cdots \oplus V_{n} \equiv V_{1}+\cdots+V_{n}$.

If in addition $V$ is normed then $V_{1} \oplus \cdots \oplus V_{n}$ will always be understood with the norm $\left\|\left(v_{1}, \ldots, v_{n}\right)\right\|=\left\|v_{1}\right\|+\cdots+\left\|v_{n}\right\|$. Then the natural map into $V$ is automatically continuous. If it is actually a homeomorphism (so in particular $\left.V_{1} \oplus \cdots \oplus V_{n} \equiv V_{1}+\cdots+V_{n}\right)$ we write $V_{1} \oplus \cdots \oplus V_{n} \equiv V_{1}+\cdots+V_{n}$ topologically. The expression $V_{1} \oplus \cdots \oplus V_{n} \equiv V_{0}$ topologically has the obvious meaning.

LeMMA 2.4. Suppose $\varnothing \neq W \subset C\left(X_{1}\right)$ and $V_{1}$ and $V_{2}$ are closed subspaces of $C\left(X_{2}\right)$ such that $V_{1} \cap V_{2}=\{0\}$. Then $\left(W \otimes V_{1}\right)^{-} \cap\left(W \otimes V_{2}\right)^{-}=\{0\}$.

Proof. Use Lemma 2.1.

Lemma 2.5. Suppose $\varnothing \neq W \subset C\left(X_{2}\right)$ and $V_{1}, \ldots, V_{n}, V$ are closed subspaces of $C\left(X_{2}\right)$ such that $V_{1} \oplus \cdots \oplus V_{n} \equiv V$. Then

(i) $V_{i_{1}}+\cdots+V_{i_{q}}$ is closed in $C\left(X_{2}\right), 1 \leqq i_{1}<\cdots<i_{q} \leqq n$.

(ii) $V_{i_{1}} \oplus \cdots \oplus V_{i_{q}} \equiv V_{i_{1}}+\cdots+V_{i_{q}}$ topologically, $1 \leqq i_{1}<\cdots<i_{q} \leqq n$.

(iii) $\left(W \otimes V_{1}\right)^{-} \oplus \cdots \oplus\left(W \otimes V_{n}\right)^{-} \equiv(W \otimes V)^{-}$topologically.

Proof. (i) and (ii) are an easy exercise using the open mapping theorem. They permit us (by induction) to reduce (iii) to the case $n=2$, the case $n=1$ being trivial.

Because $V_{1} \cap V_{2}=\{0\}$, Lemma 2.4 implies $\left(W \otimes V_{1}\right)^{-} \cap\left(W \otimes V_{2}\right)^{-}=\{0\}$. Since $\left(W \otimes V_{1}\right)^{-},\left(W \otimes V_{2}\right)^{-}$and $(W \otimes V)^{-}$are closed subspaces of $C\left(X_{1} \times X_{2}\right)$, in view of (ii) it remains only to show that $\left(W \otimes V_{1}\right)^{-}+\left(W \otimes V_{2}\right)^{-}=(W \otimes V)^{-}$. Clearly $\left(W \otimes V_{1}\right)^{-}+\left(W \otimes V_{2}\right)^{-} \subset(W \otimes V)^{-}$.

To prove the reverse inclusion, let $f \in(W \otimes V)^{-}$. We can select a sequence $\left\{f_{k}\right\}$ in $W \otimes V$ such that $\left\|f-f_{k}\right\| \rightarrow 0$. Write $f_{k}=g_{k}+h_{k}$ where $g_{k} \in W \otimes V_{1}$ and $h_{k} \in W \otimes V_{2}$.

Let $K$ be a positive constant such that $F_{1} \in V_{1}$ and $F_{2} \in V_{2}$ imply $\left\|F_{1}\right\|$ $\leqq K\left\|F_{1}+F_{2}\right\|$ and $\left\|F_{2}\right\| \leqq K\left\|F_{1}+F_{2}\right\|$; such a constant exists by (ii). We claim that $\left\|g_{j}-g_{k}\right\|,\left\|h_{j}-h_{k}\right\| \leqq K\left\|f_{j}-f_{k}\right\|$ for all $j, k$. For let $x_{1} \in X_{1}$ be given. Define $F_{1 j k}$ and $F_{2 j k}$ in $C\left(X_{2}\right)$ by $F_{1 j k}\left(x_{2}\right)=g_{j}\left(x_{1}, x_{2}\right)-g_{k}\left(x_{1}, x_{2}\right)$ and $F_{2 j k}\left(x_{2}\right)=h_{j}\left(x_{1}, x_{2}\right)$ $-h_{k}\left(x_{1}, x_{2}\right)$. Then $F_{1 j k} \in V_{1}$ and $F_{2 j k} \in V_{2}$ by Lemma 2.1, and $F_{1 j k}\left(x_{2}\right)+F_{2 j k}\left(x_{2}\right)$ $=f_{j}\left(x_{1}, x_{2}\right)-f_{k}\left(x_{1}, x_{2}\right)$. Thus $\left\|F_{1 j k}\right\|, \quad\left\|F_{2 j k}\right\| \leqq K\left\|F_{1 j k}+F_{2 j k}\right\| \leqq K\left\|f_{1}-f_{2}\right\|$, or $\left|\left(g_{j}-g_{k}\right)\left(x_{1}, x_{2}\right)\right|,\left|\left(h_{j}-h_{k}\right)\left(x_{1}, x_{2}\right)\right| \leqq K\left\|f_{1}-f_{2}\right\|$ for all $x_{2} \in X_{2}$. Letting $x_{1}$ va ry through $X_{1}$ yields $\left\|g_{j}-g_{k}\right\|,\left\|h_{j}-h_{k}\right\| \leqq K\left\|f_{j}-f_{k}\right\|$. 
Since the sequence $\left\{f_{k}\right\}$ is Cauchy, so are the sequences $\left\{g_{k}\right\}$ and $\left\{h_{k}\right\}$, hence we can find $g \in\left(W \otimes V_{1}\right)^{-}$and $h \in\left(W \otimes V_{2}\right)^{-}$such that $\left\|g-g_{k}\right\| \rightarrow 0$ and $\left\|h-h_{k}\right\|$ $\rightarrow 0$. Clearly $f=g+h$.

We now introduce polydisc algebras. The open unit disc in the complex plane will forevermore be denoted by $D$, its closure by $\bar{D}$, its bounding circle by $T$. Let $\Sigma$ be a nonempty index set and let $Z=\prod \bar{D}_{\sigma}$ be a product of $\Sigma$ copies of $\bar{D}$. Let $\bar{D}_{\sigma}$ have coordinate $z_{\sigma}$, so the typical element of $Z$ is $z=\left\{z_{\sigma}\right\}$. Let $D_{\sigma}$ and $T_{\sigma}$ be the copies of $D$ and $T$ respectively in $\bar{D}_{\sigma}$. Then $G=\prod T_{\sigma} \subset Z$. Let $\lambda$ be normalized Haar measure on $G$, i.e., the product of the normalized Lebesgue measures on the various $T_{\sigma}$. Two distinct monomials of the form $z_{\sigma_{1}}^{m_{1}} \cdots z_{\sigma_{n}}^{m_{n}}$ are then orthogonal in $L^{2}(\lambda)$.

The algebra $B$ will be $P(Z)$ the closure on $Z$ of the polynomials in the $z_{\sigma}$. Evidently $B=\otimes\left\{P\left(\bar{D}_{\sigma}\right)\right\}^{-}$and because $M_{P\left(\bar{D}_{\sigma}\right)}=\bar{D}_{\sigma}$ and $S_{P\left(\bar{D}_{\sigma}\right)}=T_{\sigma}$, it follows from Theorem 2.3 that $M_{B}=Z$ and $S_{B}=G$. We next show that analyticity characterizes the functions in $B$.

LEMMA 2.6. B consists of those $f \in C(Z)$ which are holomorphic on $D_{\sigma}$ as a function of $z_{\sigma}$ for each $\sigma \in \Sigma$.

Proof. Clearly each $f \in B$ is analytic on $D_{\sigma}$ as a function of $z_{\sigma}$. The reverse implication is well known if $\Sigma$ is finite and follows readily from, say, Osgood's lemma [GR, p. 2]. We therefore assume the lemma for finite $\Sigma$.

Suppose $f \in C(Z)$ is analytic in each variable separately. Let $\varepsilon>0$ be given; we approximate $f$ within $\varepsilon$ by a polynomial.

Using continuity of $f$, we can find a finite set $\Sigma_{0} \subset \Sigma$ such that $z^{0} \in \prod\left\{\bar{D}_{\sigma}: \sigma \in \Sigma_{0}\right)$ and $z^{1}, z^{2} \in \prod\left\{\bar{D}_{0}: \sigma \in \Sigma-\Sigma_{0}\right\}$ imply $\left|f\left(z^{0}, z^{1}\right)-f\left(z^{0}, z^{2}\right)\right|<\varepsilon / 2$. Select

$$
z^{1} \in \prod\left\{\bar{D}_{\sigma}: \sigma \in \Sigma-\Sigma_{0}\right\}
$$

and define $g \in C\left(\prod\left\{\bar{D}_{\sigma}: \sigma \in \Sigma_{0}\right\}\right)$ by $g\left(z^{0}\right)=f\left(z^{0}, z^{1}\right)$. Using the finite $-\Sigma$ version of the lemma, choose $h_{1}$ a polynomial in $\left\{z_{\sigma}: \sigma \in \Sigma_{0}\right\}$ such that $\left\|h_{1}-g\right\|<\varepsilon / 2$ in $C\left(\prod\left\{\bar{D}_{\sigma}: \sigma \in \Sigma_{0}\right\}\right)$. Define $h$ on $\prod\left\{\bar{D}_{\sigma}\right\}$ by $h\left(z^{0}, z^{2}\right)=h_{1}\left(z^{0}\right)$. Then $h$ is a polynomial and $\|h-f\|<\varepsilon$.

Let $q$ be a nonnegative integer. $H_{q}$ will denote the homogeneous polynomials of degree $q$ in the $z_{\sigma}, B_{q}$ its closure. If $F$ is a set of nonnegative integers (the symbol $F$ is henceforth reserved for this meaning), set $B_{F}=\operatorname{sp}\left(\bigcup\left\{B_{q}: q \in F\right\}\right)^{-}=$ $\operatorname{sp}\left(\bigcup\left\{H_{q}: q \in F\right\}\right)^{-}$. In particular, $B_{\{q\}}=B_{q}$.

LEMMA 2.7. Let $q$ be a nonnegative integer. Let $\left\{g_{n}\right\}$ and $\left\{h_{n}\right\}$ be sequences in $H_{q}$ and $\operatorname{sp}\left(\bigcup\left\{H_{k}: k>q\right\}\right)$ respectively such that $\left\|g_{n}+h_{n}\right\| \rightarrow 0$. Then $\left\|g_{n}\right\| \rightarrow 0$ and $\left\|h_{n}\right\| \rightarrow 0$.

Proof. Otherwise, by passing to a subsequence and normalizing if necessary, we may assume $\left\|g_{n}\right\| \rightarrow 1$ and $\left\|h_{n}\right\| \rightarrow 1$. Select a sequence $\left\{\rho_{n}\right\}$ of positive numbers such that $\rho_{n} \rightarrow 0$ and $\left\|g_{n}+h_{n}\right\| \cdot \rho_{n}^{-q} \rightarrow 0$; for instance, $\rho_{n}=\left(\left\|g_{n}+h_{n}\right\|+n^{-1}\right)^{1 / 2 q}$. 
Define $g_{n}^{\prime} \in H_{q}$ and $h_{n}^{\prime} \in \operatorname{sp}\left(\bigcup\left\{H_{k}: k>q\right\}\right)$ by $g_{n}^{\prime}(z)=g_{n}\left(\rho_{n} z\right)$ and $h_{n}^{\prime}(z)=h_{n}\left(\rho_{n} z\right)$. Since $h_{n}$ is a polynomial of total order at 0 at least $q+1$, Schwarz's lemma [GR, p. 8] yields $\left\|h_{n}^{\prime}\right\| \leqq \rho_{n}^{q+1}\left\|h_{n}\right\|$, so $\left\|h_{n}^{\prime}\right\| \cdot \rho_{n}^{-q} \rightarrow 0$. Since also $\left\|g_{n}^{\prime}+h_{n}^{\prime}\right\| \cdot \rho_{n}^{-q} \leqq\left\|g_{n}+h_{n}\right\| \cdot \rho_{n}^{-q}$ $\rightarrow 0$ we obtain $\left\|g_{n}^{\prime}\right\| \cdot \rho_{n}^{-q} \rightarrow 0$. But $g_{n}^{\prime} \cdot \rho_{n}^{-q}=g_{n}$, so $\left\|g_{n}^{\prime}\right\| \cdot \rho_{n}^{-q}=\left\|g_{n}\right\| \rightarrow 1$, a contradiction.

LEMMA 2.8. Let $q$ be a nonnegative integer and suppose $k \in F$ implies $k>q$. Then $B_{q} \oplus B_{F} \equiv B_{F \cup\{q\}}$ topologically.

Proof. Clearly $B_{q}+B_{F} \subset B_{F \cup\{q\}}$ and the remarks after Lemma 2.5 imply that $B_{q}$ is orthogonal to $B_{F}$ in $L^{2}(\lambda)$, hence $B_{q} \cap B_{F}=\{0\}$. Lemma 2.5 then reduces us to showing $B_{F \cup\{q\}} \subset B_{q}+B_{F}$.

Let $f \in B_{F \cup\{q\}}$ be given. Select sequences $\left\{g_{n}\right\} \subset H_{q}$ and $\left\{h_{n}\right\} \subset \operatorname{sp}\left(\bigcup\left\{H_{k}: k \in F\right\}\right)$ such that $\left\|f-\left(g_{n}+h_{n}\right)\right\| \rightarrow 0$. The sequence $\left\{g_{n}+h_{n}\right\}$ is Cauchy, hence by Lemma 2.7 so are the sequences $\left\{g_{n}\right\}$ and $\left\{h_{n}\right\}$. Thus we can find $g \in B_{q}$ and $h \in B_{F}$ such that $\left\|g-g_{n}\right\| \rightarrow 0$ and $\left\|h-h_{n}\right\| \rightarrow 0$. Clearly $f=g+h$.

Corollary 2.9. Let $F_{0}, F_{1}, \ldots, F_{n}$ be disjoint and $F_{1}, \ldots, F_{n}$ finite. Then $B_{F_{0}} \oplus B_{F_{1}} \oplus \cdots \oplus B_{F_{n}} \equiv B_{F_{0} \cup F_{1} \cup \cdots \cup F_{n}}$ topologically.

Let $V$ be a closed subspace of $B$, and for each nonnegative integer $q$ let $V_{q}=V \cap B_{q}$. If $F$ is any set of nonnegative integers, let $V_{F}=\operatorname{sp}\left(\bigcup\left\{V_{q}: q \in F\right\}\right)^{-}$; in particular, $V_{\{q\}}=V_{q}$. Finally, call $V$ homogeneous if $V=\operatorname{sp}\left(\bigcup\left\{V_{q}: q \geqq 0\right\}\right)^{-}$.

Similarly, if $X$ is a compact Hausdorff space and $U$ is a closed subspace of $(C(X) \otimes B)^{-}$, let $U_{q}=U \cap\left(C(X) \otimes B_{q}\right)^{-}$and $U_{F}=\operatorname{sp}\left(\bigcup\left\{U_{q}: q \in F\right\}\right)^{-}$, so $U_{\{q\}}=U_{q}$. Call $U$ homogeneous if $U=\operatorname{sp}\left(\bigcup\left\{U_{q}: q \geqq 0\right\}\right)^{-}$.

Two remarks are in order here. First, the question of whether $U$ is homogeneous depends on its representation, i.e., on the choice of $X$ and $Z$. For example, let $\bar{D}_{j}$ have coordinate $z_{j}$ for $j=1,2$. Let $W_{1} \subset P\left(\bar{D}_{1}\right)$ be the linear span of the polynomial $1+z_{1}$ and let $W_{2} \subset P\left(\bar{D}_{2}\right)$ be the linear span of the polynomial 1 , say. Let $U=$ $\left(W_{1} \otimes W_{2}\right)^{-}$. If we let $\bar{D}_{1}=X, \bar{D}_{2}=Z$ then $U$ is a homogeneous subspace of $(C(X) \otimes B)^{-}$. However, if we let $X$ be a one-point space and $Z=\bar{D}_{1} \times \bar{D}_{2}$, then we may view $U$ as a subspace of $(C(X) \otimes B)^{-}$, and $U$ is not homogeneous. In using the term, then, we shall always have a fixed representation in mind, and this representation will be clear from the context, so no ambiguity will arise. The second remark is that by letting $X$ be a one-point space, we can make facts about $(C(X) \otimes B)^{-}$imply corresponding facts about $B$.

LEMMA 2.10. For each nonnegative integer $q$ let $N_{q}$ be a subspace of $\left(C(X) \otimes B_{q}\right)^{-}$. Let $U=\operatorname{sp}\left(\bigcup\left\{N_{q}: . q \geqq 0\right\}\right)^{-}$. Then $U$ is homogeneous and $U_{q}=N_{q}^{-}$for all $q$.

Proof. Clearly $U$ is homogeneous and $N_{q} \subset U_{q}$. It remains to show that $N_{q}$ is dense in $U_{q}$. Let $f \in U_{q}$ be given. Choose sequences $\left\{g_{n}\right\} \subset N_{q}$ and

$$
\left\{h_{n}\right\} \subset \operatorname{sp}\left(\bigcup\left\{N_{k}: 0 \leqq k \neq q\right\}\right)
$$

such that $\left\|f-\left(g_{n}+h_{n}\right)\right\| \rightarrow 0$. From Corollary 2.9 and Lemma 2.5(iii) it follows that $\left\|f-g_{n}\right\| \rightarrow 0$. 
Corollary 2.11. Let $U$ and $V$ be homogeneous subspaces of $(C(X) \otimes B)^{-}$. Then $(U+V)^{-}$is a homogeneous subspace of $(C(X) \otimes B)^{-}$, and $\left((U+V)^{-}\right)_{q}$ $=\left(U_{q}+V_{q}\right)^{-}$for all $q$.

Corollary 2.12. Let $\varnothing \neq W \subset C(X)$ and let $V$ be a homogeneous subspace of $B$. Then $(W \otimes V)^{-}$is a homogeneous subspace of $(C(X) \otimes B)^{-}$, and $\left((W \otimes V)^{-}\right)_{q}$ $=\left(W \otimes V_{q}\right)^{-}$for all $q$.

Lemma 2.13. Let $F_{0}, F_{1}, \ldots, F_{n}$ be disjoint and $F_{1}, \ldots, F_{n}$ finite. Suppose $U$ is a homogeneous subspace of $(C(X) \otimes B)^{-}$. Then

topologically.

$$
U_{F_{0}} \oplus U_{F_{1}} \oplus \cdots \oplus U_{F_{n}} \equiv U_{F_{0} \cup F_{1} \cup \cdots \cup F_{n}}
$$

Proof. Corollary 2.9 and Lemma 2.5 imply that $\left(C(X) \otimes B_{F_{0}}\right)^{-} \oplus \cdots \oplus$ $\left(C(X) \otimes B_{F_{n}}\right)^{-} \equiv\left(C(X) \otimes B_{F_{0} \cup \cdots \cup F_{n}}\right)^{-}$topologically. $U_{F_{j}}$ is a closed subspace of $\left(C(X) \otimes B_{F_{j}}\right)^{-}$, so $U_{F_{0}} \oplus \cdots \oplus U_{F_{n}}$ is a closed, hence complete, subspace of $\left(C(X) \otimes B_{F_{0}}\right)^{-} \oplus \cdots \oplus\left(C(X) \otimes B_{F_{n}}\right)^{-}$. Consequently $U_{F_{0}} \oplus \cdots \oplus U_{F_{n}} \equiv U_{F_{0}}+$ $\cdots+U_{F_{n}}$ topologically, and in particular the latter is a complete, hence closed, subspace of $U_{F_{0}} \cup \cdots \cup F_{n}$. We want $U_{F_{0}}+\cdots+U_{F_{n}}=U_{F_{0}} \cup \cdots \cup F_{n}$, hence we must show that $U_{F_{0}}+\cdots+U_{F_{n}}$ is dense in $U_{F_{0} \cup \cdots \cup F_{n}}$. But this holds because $\operatorname{sp}\left(\bigcup\left\{U_{q}: q \in F_{0} \cup \cdots \cup F_{n}\right\}\right)$ is contained in $U_{F_{0}}+\cdots+U_{F_{n}}$ and is dense in $U_{F_{0}} \cup \cdots \cup F_{n}$.

3. This section is devoted to producing algebras and maximal ideals whose associated graded algebras realize preassigned structures. We obtain Theorem 3.17, the $11 \mathrm{i}: \mathrm{n}$ theorem of this paper; in fact, we obtain a slight generalization of it (Theorem 3.16).

To begin with, let $A^{\prime}$ and $B$ denote sup-norm algebras on $X=M_{A^{\prime}}$ and $Y=M_{B}$ respectively. Let $A$ be a sup-norm algebra on $X$ contained in $A^{\prime}$ and let $I$ be a closed ideal of $B$. $\mathfrak{A}$ will denote the sup-norm algebra on $X \times Y$ generated by $A^{\prime} \otimes I$ and $A \otimes B$.

Lemma 3.1. $\mathfrak{A}=\left(\left(A^{\prime} \otimes I\right)+(A \otimes B)\right)^{-} \subset\left(A^{\prime} \otimes B\right)^{-}$.

Corollary 3.2. Suppose $y \in$ hull $(I) \subset Y$. Then for each $f \in \mathfrak{A}$ the function $f_{y} \in C(X)$ given by $f_{y}(x)=f(x, y)$ is in $A$.

Let $\tau: A \rightarrow \mathfrak{A}$ and $\eta: B \rightarrow \mathfrak{A}$ be the isometric isomorphisms $\tau(a)=a \otimes 1$ and $\eta(b)=1 \otimes b$. The dual maps are $\tau_{*}: M_{\mathfrak{A}} \rightarrow M_{A}$ and $\eta_{*}: M_{\mathfrak{U}} \rightarrow M_{B}=Y$. Define $\pi: M_{\mathfrak{U}} \rightarrow M_{A} \times Y$ by $\pi(\varphi)=\left(\tau_{*}(\varphi), \eta_{*}(\varphi)\right)$.

THEOREM 3.3. $\pi$ maps $M_{\mathfrak{U}}$ homeomorphically onto $\left(M_{A} \times\right.$ hull $\left.(I)\right) \cup(X \times Y)$.

Proof. Clearly $\pi$ is continuous and $\pi\left(M_{\mathfrak{U}}\right) \supset X \times Y$.

Let $(\alpha, y) \in M_{A} \times$ hull $(I)$ be given. The map $f \rightarrow f_{y}$ is a homomorphism of $\mathfrak{A}$ onto $A$ (see Corollary 3.2). Define $\varphi \in M_{\mathfrak{A}}$ by $\varphi(f)=\alpha\left(f_{y}\right)$. Then $\pi(\varphi)=(\alpha, y)$.

Therefore $\pi\left(M_{\mathfrak{A}}\right) \supset\left(M_{A} \times\right.$ hull $\left.(I)\right) \cup(X \times Y)$. 
Now suppose $\varphi \in M_{\mathfrak{A}}$ and $\pi(\varphi)=(\alpha, y) \in M_{A} \times Y$. We must show that $(\alpha, y)$ $\in\left(M_{A} \times\right.$ hull $\left.(I)\right) \cup(X \times Y)$ and that $\varphi$ is uniquely determined by $(\alpha, y)$.

Case 1. $y \in$ hull $(I)$. It suffices to show that $\varphi \mid\left(A^{\prime} \otimes I\right)=0$ and $\varphi(a \otimes b)=\alpha(a) b(y)$ for $a \in A, b \in B$.

If $a \in A^{\prime}$ and $i \in I$ then $(\varphi(a \otimes i))^{2}=\varphi\left(a^{2} \otimes i^{2}\right)=\varphi\left(a^{2} \otimes i\right) \cdot \varphi(1 \otimes i)=\varphi\left(a^{2} \otimes i\right)$ $\cdot i(y)=0$, so $\varphi(a \otimes i)=0$. Thus $\varphi \mid\left(A^{\prime} \otimes I\right)=0$.

If $a \in A$ and $b \in B$ then

$$
\varphi(a \otimes b)=\varphi(a \otimes 1) \cdot \varphi(1 \otimes b)=\alpha(a) b(y) .
$$

Case 2. $y \notin$ hull $(I)$. We will show that $\alpha$ is evaluation at some $x \in X$, and that $\varphi\left(a^{\prime} \otimes i\right)=a^{\prime}(x) i(y)$ and $\varphi(a \otimes b)=a(x) b(y)$ whenever $a \in A, a^{\prime} \in A^{\prime}, b \in B, i \in I$.

Select $i_{0} \in I$ such that $i_{0}(y)=1$. Define $L \in A^{\prime *}$ by $L\left(a^{\prime}\right)=\varphi\left(a^{\prime} \otimes i_{0}\right)$. Then $L(1)=1$, so $L \neq 0$. Further, $L\left(a_{1}^{\prime} \cdot a_{2}^{\prime}\right)=\varphi\left(a_{1}^{\prime} \cdot a_{2}^{\prime} \otimes i_{0}\right)=\varphi\left(a_{1}^{\prime} \cdot a_{2}^{\prime} \otimes i_{0}\right) \cdot \varphi\left(1 \otimes i_{0}\right)$ $=\varphi\left(a_{1}^{\prime} \otimes i_{0}\right) \cdot \varphi\left(a_{2}^{\prime} \otimes i_{0}\right)=L\left(a_{1}^{\prime}\right) \cdot L\left(a_{2}^{\prime}\right)$, so $L$ is multiplicative. Thus $L$ is evaluation at some $x \in X$. If $a \in A$ then $\alpha(a)=\varphi(a \otimes 1) \cdot \varphi\left(1 \otimes i_{0}\right)=\varphi\left(a \otimes i_{0}\right)=L(a)=a(x)$, so $\alpha$ is evaluation at $x$.

If $a \in A, b \in B$ then $\varphi(a \otimes b)=\varphi(a \otimes 1) \cdot \varphi(1 \otimes b)=\alpha(a) b(y)=a(x) b(y)$. If $a^{\prime} \in A^{\prime}$, $i \in I$ then $\varphi\left(a^{\prime} \otimes i\right)=\varphi\left(a^{\prime} \otimes i\right) \cdot \varphi\left(1 \otimes i_{0}\right)=\varphi\left(a^{\prime} \otimes i_{0}\right) \cdot \varphi(1 \otimes i)=L\left(a^{\prime}\right) i(y)=a^{\prime}(x) i(y)$.

Lemma 3.4. Suppose $y \in S_{B}-$ hull $(I)$ and $V$ is an open set in $Y$ containing $y$. Then we can find $i \in I$ such that $\|i\|=1$ and $\|i \mid Y-V\|<1$.

Proposition 3.5. $\left(S_{A^{\prime}} \times\left(S_{B}-\text { hull }(I)\right)\right)^{-} \cup\left(S_{A} \times S_{B}\right) \subset S_{\mathfrak{U}} \subset S_{A^{\prime}} \times S_{B}$.

CoROLlaRY 3.6. If int $_{S_{B}}$ (hull $\left.(I)\right)=\varnothing$, then $S_{\mathfrak{U}}=S_{A^{\prime}} \times S_{B}$.

Now let $Z$ and $B$ be as in the discussion preceding Lemma 2.6, i.e., $Z=\prod\left\{\bar{D}_{\sigma}: \sigma \in \Sigma\right\}$ is a product of closed discs and $B=P(Z) ; Z$ and $B$ will serve as the $Y$ and $B$ of our construction. $I$ will denote a proper homogeneous ideal in $B$, that is, a proper homogeneous subspace which is an ideal in $B$; this is not to be confused with the term "homogeneous ideal" used earlier in connection with polynomial rings. $A$ and $A^{\prime}$ will be sup-norm algebras on $X=M_{A^{\prime}}$ with $A \subset A^{\prime}$ and we will construct $\mathfrak{A}$. Observe that $0 \in$ hull $(I)$, so by Theorem $3.3 M_{A} \times\{0\} \subset M_{\mathfrak{A}}$.

We begin by describing the permissible $I$.

LEMMA 3.7. Let there be given a family $\left\{J_{q}: q \geqq 0\right\}$ of subspaces of $B$ such that $J_{0}=\{0\}, J_{q} \subset B_{q}$, and $z_{\sigma} J_{q} \subset J_{q+1}$ for each $\sigma \in \Sigma$ and $q \geqq 0$. Then $I=\operatorname{sp}\left(\bigcup\left\{J_{q}: q \geqq 0\right\}\right)^{-}$ is a proper homogeneous ideal in $B$ and $I_{q}=J_{q}^{-}$for all $q \geqq 0$.

Our next objective is an explicit description of $\left(\mathfrak{A}_{\varphi}^{n}\right)^{-}$for certain $\varphi \in M_{\mathfrak{A}}$.

LEMMA 3.8. $\mathfrak{A}$ is a homogeneous subspace of $(C(X) \otimes B)^{-}$, and $\mathfrak{A}_{q}=\left(\left(A^{\prime} \otimes I_{q}\right)\right.$ $\left.+\left(A \otimes B_{q}\right)\right)^{-}$for all $q \geqq 0$.

Proof. Use Corollary 2.12 to obtain such a result for $\left(A^{\prime} \otimes I\right)^{-}$and $(A \otimes B)^{-}$, and then use Lemma 3.1 and Corollary 2.11 to obtain the desired statement.

Let $\varphi_{0} \in M_{A}-X$ be given and set $\varphi=\left(\varphi_{0}, 0\right) \in M_{\mathfrak{A}}$. 
TheOREM 3.9. $\left(\mathfrak{A}_{\varphi}^{n}\right)^{-}$is the closed linear span of $A^{\prime} \otimes I$, the spaces $A \otimes B_{q}$ for $q \geqq n$, and the spaces $A_{\varphi_{0}}^{n-q} \otimes B_{q}$ for $0 \leqq q<n$.

Proof. Lemma 3.8 proves the theorem for $n=0$. We next prove the theorem for $n=1$. $\mathfrak{A}_{\varphi}$ obviously contains $A^{\prime} \otimes I, A_{\varphi_{0}} \otimes B_{0}$ and $A \otimes B_{q}$ for $q \geqq 1$. Conversely, let $f \in \mathfrak{A}_{\varphi}$. Select a sequence $\left\{f_{k}\right\}$ in $\mathfrak{A}_{\varphi}$ such that $\left\|f-f_{k}\right\| \rightarrow 0$ and $f_{k}=s_{k}+t_{k}+u_{k}$ where $s_{k} \in A^{\prime} \otimes I, t_{k} \in \operatorname{sp}\left(\bigcup\left\{A \otimes B_{q}: q>0\right\}\right)$ and $u_{k} \in A \otimes B_{0}$; this is possible by Lemma 3.8. Then $\varphi\left(f_{k}\right)=\varphi\left(s_{k}\right)=\varphi\left(t_{k}\right)=0$, so $\varphi\left(u_{k}\right)=0$, hence $u_{k} \in A_{\varphi_{0}} \otimes B_{0}$. This proves the theorem for $n=1$.

Suppose $N>1$; we prove the theorem for $n=N$.

Let $a^{\prime} \in A^{\prime}$ be given. Since hull $\left(A_{\varphi_{0}}^{N}\right)=\left\{\varphi_{0}\right\}$ and $\varphi_{0} \notin X, A_{\varphi_{0}}^{N}$ generates the unit ideal in $A^{\prime}$, so we can find $a_{1}, \ldots, a_{m}$ in $A_{\varphi_{0}}^{N}$ and $a_{1}^{\prime}, \ldots, a_{m}^{\prime}$ in $A^{\prime}$ such that $a^{\prime}=a_{1} a_{1}^{\prime}+\cdots+a_{m} a_{m}^{\prime}$. If $i \in I$ then

$$
a^{\prime} \otimes i=\left(a_{1} \otimes 1\right)\left(a_{1}^{\prime} \otimes i\right)+\cdots+\left(a_{m} \otimes 1\right)\left(a_{m}^{\prime} \otimes i\right) \in \mathfrak{H}_{\varphi}^{N} .
$$

Thus $A^{\prime} \otimes I \subset \mathfrak{A}_{\varphi}^{N}$.

Next, observe that for $q>0, B_{q} \subset\left(B_{1}^{q}\right)^{-}$; hence for $q \geqq 0,1 \otimes B_{q} \subset\left(\mathfrak{U}_{\varphi}^{q}\right)^{-}$. Also, $A_{\varphi_{0}}^{p} \otimes 1 \subset \mathfrak{A}_{\varphi^{p}}^{p}$. Therefore $A_{\varphi_{0}}^{p} \otimes B_{q} \subset \operatorname{sp}\left(\left(A_{\varphi_{0}}^{p} \otimes 1\right)\left(1 \otimes B_{q}\right)\right) \subset\left(\mathfrak{H}_{\varphi}^{p+q}\right)^{-}$. This leads to $A \otimes B_{q} \subset\left(\mathfrak{H}_{\varphi}^{N}\right)^{-}$if $q \geqq N$ and $A_{\varphi_{0}^{N}}^{N-q} \otimes B_{q} \subset\left(\mathfrak{U}_{\varphi}^{N}\right)^{-}$if $q<N$. Thus $\left(\mathfrak{R}_{\varphi}^{N}\right)^{-}$contains the desired linear span.

For the reverse inclusion, it suffices to show that if $f_{1}, \ldots, f_{N}$ are in $\mathfrak{A}_{\varphi}$ then $f_{1} \cdots f_{N}$ is in the desired closed linear span. The case $n=1$ of the theorem implies that we may assume that each $f_{j}$ is in $\left(A^{\prime} \otimes I\right)+\left(A_{\varphi_{0}} \otimes B_{0}\right)+\operatorname{sp}\left(\bigcup\left\{A \otimes B_{q}: q>0\right\}\right)$, and applying Corollary 2.12 to $A^{\prime} \otimes I$ we see that we may suppose that

$$
f_{j} \in \mathrm{sp}\left(\bigcup\left\{A^{\prime} \otimes I_{q}: q>0\right\}\right)+\left(A_{\varphi_{0}} \otimes B_{0}\right)+\operatorname{sp}\left(\bigcup\left\{A \otimes B_{q}: q>0\right\}\right) .
$$

But under these conditions $f_{1} \cdots f_{N}$ is a finite sum of terms of the form

$$
u=\left(\alpha_{1} \otimes \beta_{1}\right) \cdots\left(\alpha_{N_{1}} \otimes \beta_{N_{1}}\right)\left(a_{1}^{\prime} \otimes i_{1}\right) \cdots\left(a_{N_{2}}^{\prime} \otimes i_{N_{2}}\right)\left(a_{1} \otimes b_{1}\right) \cdots\left(a_{N_{3}} \otimes b_{N_{3}}\right)
$$

with $\alpha_{r} \in A_{\varphi_{0}}, \beta_{r} \in B_{0}, a_{s}^{\prime} \in A^{\prime}, i_{s} \in I_{p_{s}}$ for some $p_{s} \geqq 1, a_{t} \in A$ and $b_{t} \in B_{q_{t}}$ for some $q_{t} \geqq 1$ with $N_{1}+N_{2}+N_{3}=N$. If $N_{2} \neq 0, u \in A^{\prime} \otimes I$. If $N_{2}=0$ and $N_{1} \neq 0$, set $q=N-N_{1}<N$; then $u \in A_{\varphi_{0}}^{N-q} \otimes B_{q}$. If $N_{1}=N_{2}=0$ then $N_{3}=N$ and $u \in A \otimes B_{q}$ for a $q \geqq N$. Thus each such $u$ is in the desired closed linear span, hence so is $f_{1} \cdots f_{N}$.

COROLlary 3.10. $\left(\mathfrak{A}_{\varphi}^{n}\right)^{-}$is a homogeneous subspace of $(C(X) \otimes B)^{-}$and $\left(\left(\Re_{\varphi}^{n}\right)^{-}\right)_{q}=\left(\left(A^{\prime} \otimes I_{q}\right)+\left(A \otimes B_{q}\right)\right)^{-}$if $q \geqq n$, while $\left(\left(\mathfrak{A}_{\varphi}^{n}\right)^{-}\right)_{q}=\left(\left(A^{\prime} \otimes I_{q}\right)+\left(A_{\varphi_{0}}^{n-q} \otimes B_{q}\right)\right)^{-}$ if $0 \leqq q<n$.

Proof. Use Theorem 3.9, Lemma 2.10, and Corollaries 2.11 and 2.12.

We now associate to $\mathfrak{A}$ and $\varphi$ the complex of objects in $\S 1$, i.e., the graded algebra $V$, the maps $l_{n}$, the ideal $U$ and the quotient graded algebra $Q$.

Let $\mathscr{B}=\operatorname{sp}\left(\bigcup\left\{B_{n}: n \geqq 0\right\}\right) \subset B$ and $\Lambda=\operatorname{sp}\left(\bigcup\left\{I_{n}: n \geqq 0\right\}\right) \subset \mathscr{B}$. $\mathscr{B}$ has an obvious graded algebra structure, in which $\Lambda$ is a homogeneous ideal. Define a linear map 
$L: \mathscr{B} \rightarrow V$ by extending linearly the map $L(b)=l_{n}(1 \otimes b)$ for $b \in B_{n} . L$ is a graded algebra homomorphism of $\mathscr{B}$ into $V$. By composition with the quotient map we obtain a graded algebra homomorphism $L^{*}: \mathscr{B} \rightarrow Q$.

LEMMA 3.11. $L\left(I_{n}\right) \subset U_{n}$ for all $n$. Equivalently, $L^{*} \mid \Lambda=0$.

Proof. $1 \otimes I_{n} \subset A^{\prime} \otimes I \subset\left(\mathfrak{A}_{\varphi}^{n+1}\right)^{-}$by Theorem 3.9, hence $L\left(I_{n}\right)=l_{n}\left(1 \otimes I_{n}\right)$ $\subset l_{n}\left(\left(\mathfrak{A}_{\varphi}^{n+1}\right)^{-}\right)=U_{n}$.

Thus $L^{*}$ determines a graded algebra homomorphism $\tilde{L}: \mathscr{B} \mid \Lambda \rightarrow Q$ where $\mathscr{B} \mid \Lambda=\sum_{n=0}^{\infty} \oplus(\mathscr{B} \mid \Lambda)_{n}$ with $(\mathscr{B} / \Lambda)_{n}=B_{n} / I_{n}$. Our next objective will be to obtain conditions under which $\tilde{L}$ is an isomorphism into $Q$, that is, under which $\operatorname{ker}\left(L^{*}\right)=\Lambda$. The key lies in the following definition: the homogeneous subspace $W$ of $B$ will be called complemented if for each $q \geqq 0$ there exists $J_{q}$ a closed subspace of $B_{q}$ such that $W_{q} \oplus J_{q} \equiv B_{q}$ topologically. Note that if $\Sigma$ is finite, that is, if $B$ consists of analytic functions in finitely many variables, then any homogeneous subspace of $B$ is automatically complemented. $\left\{J_{q}\right\}$ is called a complement for $W$.

LEMMA 3.12. If $I$ is complemented and $\left\{J_{q}\right\}$ is a complement for $I$, then $\left(A^{\prime} \otimes I_{q}\right)^{-}$ $\oplus\left(A \otimes J_{q}\right)^{-} \equiv\left(\left(\mathfrak{Q}_{\varphi}^{n}\right)^{-}\right)_{q}$ topologically if $q \geqq n$, and $\left(A^{\prime} \otimes I_{q}\right)^{-} \oplus\left(A_{\varphi_{0}}^{n-q} \otimes J_{q}\right)^{-}$ $\equiv\left(\left(\mathfrak{A}_{\varphi}^{n}\right)^{-}\right)_{q}$ topologically if $0 \leqq q<n$.

Proof. Lemma 2.5 (iii) leads to $\left(A^{\prime} \otimes I_{q}\right)^{-} \oplus\left(A_{\varphi_{0}}^{p} \otimes J_{q}\right)^{-} \equiv\left(A^{\prime} \otimes I_{q}\right)^{-}+$ $\left(A_{\varphi_{0}}^{p} \otimes J_{q}\right)^{-}$topologically, so the latter is a closed subspace of $(C(X) \otimes B)^{-}$. In view of Corollary 3.10 and the fact (which also follows from Lemma 2.5 (iii)) that $\left(A_{\varphi_{0}}^{p} \otimes B_{q}\right)^{-}=\left(A_{\varphi_{0}}^{p} \otimes I_{q}\right)^{-}+\left(A_{\varphi_{0}}^{p} \otimes J_{q}\right)^{-}$we obtain the lemma.

Proposition 3.13. If $I$ is complemented then $\Lambda=\operatorname{ker}\left(L^{*}\right)$.

Proof. We know (Lemma 3.11) that $\Lambda \subset \operatorname{ker}\left(L^{*}\right)$. Since $\operatorname{ker}\left(L^{*}\right)$ is a homogeneous ideal in $\mathscr{B}$, we have only to show that $\operatorname{ker}\left(L^{*}\right) \cap B_{n} \subset I_{n}$.

Suppose $b \in \operatorname{ker}\left(L^{*}\right) \cap B_{n}$. Write $b=b_{1}+b_{2}$ where $b_{1} \in I_{n}$ and $b_{2} \in J_{n}$; here $\left\{J_{q}\right\}$ is a complement for $I$. We must show that $b_{2}=0$.

$L^{*}(b)=0$ implies $1 \otimes b \in\left(\left(\mathfrak{H}_{\varphi}^{n+1}\right)^{-}\right)_{n}$, and Lemma 3.12 yields a $g \in\left(A^{\prime} \otimes I_{n}\right)^{-}$ and $h \in\left(A_{\varphi_{0}} \otimes J_{n}\right)^{-}$such that $1 \otimes b=-g+h$. Then $\left(\left(1 \otimes b_{1}\right)-g\right) \in\left(C(X) \otimes I_{n}\right)^{-}$ and $\left(h-\left(1 \otimes b_{2}\right)\right) \in\left(C(X) \otimes J_{n}\right)^{-}$and $\left(1 \otimes b_{1}\right)-g=h-\left(1 \otimes b_{2}\right)$, so Lemma 2.4 implies $h=1 \otimes b_{2}$. Since $h$ and $1 \otimes b_{2}$ are in $A \otimes B$, we may evaluate them at $\left(\varphi_{0}, y\right)$ for each $y \in Z$, and we obtain $b_{2}(y)=\left(1 \otimes b_{2}\right)^{\wedge}\left(\varphi_{0}, y\right)=\hat{h}\left(\varphi_{0}, y\right)=0$, the last equality because $h \in\left(A_{\varphi_{0}} \otimes J_{n}\right)^{-}$. Thus $b_{2}=0$.

Thus if $I$ is complemented then $\tilde{L}$ is an isomorphism into $Q$. It remains to determine the size of the image of $\tilde{L}$; this will lead to a reasonable condition on $A$ which guarantees that $\tilde{L}$ be onto $Q$.

LEMMA 3.14. If $I$ is compleminted and $\left\{J_{q}\right\}$ is a complement for $I$, there exist natural isomorphisms $\nu_{n}$ of $\sum_{q=0}^{n} \oplus\left(\left(A_{\varphi_{0}}^{n-q} \otimes J_{q}\right)^{-} /\left(A_{\varphi_{0}}^{n+1-q} \otimes J_{q}\right)^{-}\right)$onto $Q_{n}$ such that $\tilde{L}\left((\mathscr{B} \mid \Lambda)_{n}\right)=\nu_{n}\left(\left(A \otimes J_{n}\right)^{-} /\left(A_{\varphi_{0}} \otimes J_{n}\right)^{-}\right)$. 
Proof. Let $F(n)$ consist of all positive integers strictly greater than $n$. From Corollary 3.10 and Lemma 2.13 we see that $\left(\left(\mathfrak{U}_{\varphi}^{n}\right)^{-}\right)_{0} \oplus \cdots \oplus\left(\left(\mathfrak{U}_{\varphi}^{n}\right)^{-}\right)_{n} \oplus\left(\left(\mathfrak{U}_{\varphi}^{n}\right)^{-}\right)_{F(n)}$ $\left.\equiv\left(\mathfrak{Q}_{\varphi}^{n}\right)^{n}\right)^{-}$topologically while

$$
\left(\left(\mathfrak{A}_{\varphi}^{n+1}\right)^{-}\right)_{0} \oplus \cdots \oplus\left(\left(\mathfrak{U}_{\varphi}^{n+1}\right)^{-}\right)_{n} \oplus\left(\left(\mathfrak{A}_{\varphi}^{n+1}\right)^{-}\right)_{F(n)} \equiv\left(\mathfrak{A}_{\varphi}^{n+1}\right)^{-}
$$

topologically, where the second direct sum is imbedded in the first in an obvious fashion. Lemma 3.12 yields $\left(\left(\mathfrak{Q}_{\varphi}^{n}\right)^{-}\right)_{F(n)}=\left(\left(\mathfrak{N}_{\varphi}^{n+1}\right)^{-}\right)_{F(n)}$ and, for $0 \leqq q \leqq n,\left(A^{\prime} \otimes I_{q}\right)^{-}$ $\oplus\left(A_{\varphi_{0}}^{n-q} \otimes J_{q}\right)^{-} \equiv\left(\left(\mathfrak{N}_{\varphi}^{n}\right)^{-}\right)_{q} \quad$ topologically $\quad$ while $\quad\left(A^{\prime} \otimes I_{q}\right)^{-} \oplus\left(A_{\varphi_{0}}^{n+1-q} \otimes J_{q}\right)^{-}$ $\equiv\left(\left(\mathfrak{A}_{\Phi}^{n+1}\right)^{-}\right)_{q}$ topologically. The map $\nu_{n}$ is then defined by letting

$$
\nu_{n}\left(f+\left(A_{\varphi_{0}}^{n+1-q} \otimes J_{q}\right)^{-}\right)=l_{n}(f)+U_{n}
$$

for $f \in\left(A_{\varphi_{0}}^{n-q} \otimes J_{q}\right)^{-}$and $0 \leqq q \leqq n$. Clearly $\nu_{n}$ is an isomorphism of the given direct sum onto $Q_{n}$. It remains to prove that $L^{*}\left(B_{n}\right)=v_{n}\left(\left(A \otimes J_{n}\right)^{-} /\left(A_{\varphi_{0}} \otimes J_{n}\right)^{-}\right)$.

If $b \in I_{n}$ then $L^{*}(b)=0$ by Lemma 3.11. If $b \in J_{n}$ then $L(b) \in l_{n}\left(\left(A \otimes J_{n}\right)^{-}\right)$so $L^{*}(b)=v_{n}\left(l_{n}^{-1}(L(b))+\left(A_{\varphi_{0}} \otimes J_{n}\right)^{-}\right)$. Thus $L^{*}\left(B_{n}\right) \subset v_{n}\left(\left(A \otimes J_{n}\right)^{-} /\left(A_{\varphi_{0}} \otimes J_{n}\right)^{-}\right)$.

For the reverse inclusion, let $f \in\left(A \otimes J_{n}\right)^{-}$be given. Then $f \in(A \otimes B)^{-}$, so we can define $b \in J_{n}$ by $b(y)=\hat{f}\left(\varphi_{0}, y\right)$ for $y \in Z$. Then $1 \otimes b \in\left(A \otimes J_{n}\right)^{-}$and $\hat{f}\left(\varphi_{0}, y\right)$ $=(1 \otimes b)^{\wedge}\left(\varphi_{0}, y\right)$ for all $y \in Z$.

Since $A_{\varphi_{0}} \oplus \boldsymbol{C} \equiv A$ topologically, Lemma 2.5 yields $\left(A_{\varphi_{0}} \otimes J_{n}\right)^{-} \oplus\left(\boldsymbol{C} \otimes J_{n}\right)^{-}$ $\equiv\left(A \otimes J_{n}\right)^{-}$topologically, so we can write $f-(1 \otimes b)=g+h$ where $g \in\left(A_{\varphi_{0}} \otimes J_{n}\right)^{-}$ and $h \in\left(\boldsymbol{C} \otimes J_{n}\right)^{-}=1 \otimes J_{n}$. Then $\hat{g}\left(\varphi_{0}, y\right)=0$ and therefore $\hat{h}\left(\varphi_{0}, y\right)=0$ for all $y \in Z$. But $\hat{h}\left(\varphi_{0}, y\right)=u(y)$ where $h=1 \otimes u$, so $h=0$ and $f-(1 \otimes b)=g \in\left(A_{\varphi_{0}} \otimes J_{n}\right)^{-}$. Thus $L^{*}(b)=l_{n}(1 \otimes b)+U_{n}=l_{n}(f-g)+U_{n}=l_{n}(f)+U_{n}=v_{n}\left(f+\left(A_{\omega_{0}} \otimes J_{n}\right)^{-}\right)$. This completes the proof.

CoRollary 3.15. If I is complemented, then in order that $\tilde{L}$ be onto $Q$ it is necessary and sufficient that $A_{\varphi_{0}}=\left(A_{\varphi_{0}}^{2}\right)^{-}$.

Proof. From Lemma 3.14 we see that $A_{\varphi_{0}}=\left(A_{\varphi_{0}}^{2}\right)^{-}$implies that $\tilde{L}$ is onto, and conversely that if $\tilde{L}$ is onto then $\left(A_{\varphi_{0}} \otimes J_{0}\right)^{-}=\left(A_{\varphi_{0}}^{2} \otimes J_{0}\right)^{-}$. Because $I$ is a proper ideal in $B$ we have $J_{0}=B_{0}=C$, so $\left(A_{\varphi_{0}} \otimes J_{0}\right)^{-}=\left(A_{\varphi_{0}}^{2} \otimes J_{0}\right)^{-}$implies $A_{\varphi_{0}}=\left(A_{\varphi_{0}}^{2}\right)^{-}$.

We shall now select $X, A, A^{\prime}$ and $\varphi_{0}$ to make $A_{\varphi_{0}}=\left(A_{\varphi_{0}}^{2}\right)^{-} . X$ will denote a torus $T^{2}$ and $A^{\prime}=C(X)$. Let $\mu$ denote normalized Lebesgue measure on $T^{2}$ and let $\alpha$ be a positive irrational number. Let

$$
A=\left\{f \in C(X): \int_{T^{2}} f\left(x_{1}, x_{2}\right) x_{1}^{k_{1}} x_{2}^{k_{2}} d \mu\left(x_{1}, x_{2}\right)=0 \text { whenever } k_{1}+k_{2} \alpha>0\right\} .
$$

In [W1] $A$ is discussed; it is revealed that $A$ is a Dirichlet algebra on $X$ and that $\varphi_{0} \in A^{*}$ defined by $\varphi_{0}(f)=\int_{T^{2}} f d \mu$ is a member of $M_{A}-X$ which is a one-point part for $A$. Standard Fourier series arguments show that $A$ is the closed linear span of the functions $x_{1}^{k_{1}} x_{2}^{k_{2}}$ for $k_{1}+k_{2} \alpha \geqq 0$, from which it is easy to show directly that $A_{\varphi_{0}}=\left(A_{\varphi_{0}}^{2}\right)^{-}$; this in fact implies that $\varphi_{0}$ is a one-point part (see [S1]). Alternatively, starting with the assertion that $\varphi_{0}$ is a one-point part, we can conclude from [S1] that $A_{\varphi_{0}}=\left(A_{\varphi_{0}}^{2}\right)^{-}$. 
THEOREM 3.16. If I is a complemented homogeneous ideal in $B$ then there exists a sup-norm algebra $\mathfrak{A}$ and $a \varphi \in M_{\mathfrak{A}}$ such that if $Q, \mathscr{B}$ and $\Lambda$ have the meanings stated before Lemma 3.11 then $\mathscr{B} / \Lambda$ and $Q$ are isomorphic as graded algebras.

Proof. If $I=B$ the theorem is trivial (and can be realized with a one-dimensional algebra). If $I$ is a proper ideal, let $X, A, A^{\prime}$ and $\varphi_{0}$ be as above and construct $\mathfrak{A}$ on $X \times Z$ in the usual manner. Construct $L, L^{*}$ and $\tilde{L}$ as usual. By Proposition 3.13 $\tilde{L}$ is an isomorphism of $\mathscr{B} / \Lambda$ into $Q$, and by Corollary $3.15 \tilde{L}$ maps $\mathscr{B} / \Lambda$ onto $Q$.

THEOREM 3.17. Let $R=C\left[X_{1}, \ldots, X_{r}\right]$ be the graded polynomial algebra in $r<\infty$ variables. Let $J$ be a homogeneous ideal (algebraic sense) in $R$. Then there exists a sup-norm algebra $\mathfrak{A}$ and $a \varphi \in M_{\mathfrak{A}}$ such that if $Q$ has the meaning stated before Lemma 3.11 then $R / J$ and $Q$ are isomorphic as graded algebras.

Proof. Let $\Sigma=\{1, \ldots, r\}$ and let $\gamma: R \rightarrow B$ be the obvious imbedding. Let $I=$ $\gamma(J)^{-}$, so by Lemma $3.9 I$ is a homogeneous ideal in $B$ and $I_{n}=\gamma\left(J_{n}\right) . I$ is complemented, so Theorem 3.16 enables us to construct $\mathfrak{A}$ and $\varphi$ such that $\mathscr{B} / \Lambda$ and $Q$ are isomorphic as graded algebras. Finally, note that $\mathscr{B} / \Lambda$ and $R / J$ are naturally isomorphic as graded algebras.

4. It is a bonus of our construction that the algebra constructed in the proof of Theorem 3.17 is actually a local algebra. This section will be devoted for the most part to proving this fact.

We begin with some terminology; for extensive discussion of these notions see [R]. Let $A$ be a sup-norm algebra on $X$ and let $f \in C(X)$. $f$ is locally in $A$ on $X$ [resp., A-holomorphic on $X$ ] if there is an open covering $\left\{U_{\alpha}\right\}$ of $X$ (which may of course be taken finite) such that $f\left|U_{\alpha} \in A\right| U_{\alpha}$ [resp., $f \mid U_{\alpha} \in\left(A \mid U_{\alpha}\right)^{-}$]. $A$ is called local [resp., holomorphically closed] on $X$ if whenever $f$ is locally in $A$ [resp., $A$ holomorphic] on $X$, we have $f \in A$.

A local maximum modulus theorem holds for $A$-holomorphic functions [R, Theorem 2.2] and leads to the fact that if $A$ is local [resp., holomorphically closed] on $X$, then $A$ is local [resp., holomorphically closed] on $M_{A}$. It will also be useful to know that if $X$ is connected and $A$ is analytic on $X$ (i.e., $f \in A$ and int $_{X} f^{-1}(0) \neq \varnothing$ imply $f=0$ ) then $A$ is local on $X$; a trivial argument proves this.

Assume $\Sigma$ is finite, say $\Sigma=\{1, \ldots, r\}$. Let $I$ be a proper homogeneous ideal in $B$. $I$ is automatically complemented ; further, letting $J_{q}$ be the orthogonal complement of $I_{q}$ in $B_{q}$ (where $I_{q}, J_{q}$ and $B_{q}$ are thought of as subspaces of $L^{2}(\lambda), \lambda$ being normalized Lebesgue measure on $\left.G=T_{1} \times \cdots \times T_{r} \subset Z\right)$, we see that $\left\{J_{q}\right\}$ is a complement for $I$ and $\operatorname{sp}\left(\bigcup\left\{J_{q}: q \geqq 0\right\}\right)^{-}$is orthogonal to $I$ in $L^{2}(\lambda)$.

If $f \in C(X \times Z)$ we can define a number of associated functions.

First, for each $g \in B$ define $f_{g} \in C(X)$ by $f_{g}(x)=\int_{G} g(z)^{-} f(x, z) d \lambda(z)$. Observe that $\left\|f_{g}\right\| \leqq\|g\| \cdot\|f\|$.

Second, for each nonnegative integer $q$ and each $x \in X, f_{q}^{x}$ will denote the orthogonal projection into $B_{q}$ of the function $z \rightarrow f(x, z)$. If $e_{q, 1}, \ldots, e_{q, N(q)}$ is the 
usual orthonormal basis of $B_{q}$ consisting of the monic monomials of degree $q$-so $N(q)=C_{q+r-1, q} \leqq(q+r-1)^{r-1}$-then $f_{q}^{x}=f_{e_{q, 1}}(x) e_{q, 1}+\cdots+f_{e_{q, N(q)}}(x) e_{q, N(q)}$ and therefore $\left\|f_{q}^{x}\right\| \leqq\left\|f_{e_{q, 1}}\right\|\left\|e_{q, 1}\right\|+\cdots+\left\|f_{e_{q, N(q)}}\right\|\left\|e_{q, N(q)}\right\| \leqq N(q)\|f\| \leqq(q+r-1)^{r-1}\|f\|$ for all $x \in X$.

Finally, if $0 \leqq t<1$ define $f^{t} \in C(X \times Z)$ by $f^{t}(x, z)=f(x, t z)$.

LEMMA 4.1. If $f$ is analytic on $D_{\sigma}$ as a function of $z_{\sigma}$ for each $\sigma \in \Sigma$, then

$$
\lim _{K \rightarrow \infty}\left(\sup \left\{\left|f^{t}(x, z)-\sum_{q=0}^{K} f_{q}^{x}(z) t^{q}\right|:(x, z) \in X \times Z\right\}\right)=0 .
$$

Proof. $\sum_{q=0}^{\infty} f_{q}^{x}$ is the power series expansion of $z \rightarrow f(x, z)$, so $x \in X$ and $z \in D_{1} \times \cdots \times D_{r}$ imply $f(x, z)=\lim _{K \rightarrow \infty} \sum_{q=0}^{K} f_{q}^{x}(z)$. Since $f_{q}^{x}(t z)=f_{q}^{x}(z) t^{q}$ we obtain

$$
\begin{aligned}
\left|f^{t}(x, z)-\sum_{q=0}^{K} f_{q}^{x}(z) t^{q}\right| & =\left|\sum_{q=K+1}^{\infty} f_{q}^{x}(z) t^{q}\right| \\
& \leqq \sum_{q=K+1}^{\infty}\left\|f_{q}^{x}\right\| t^{q} \leqq \sum_{q=K+1}^{\infty}(q+r-1)^{r-1}\|f\| t^{q} .
\end{aligned}
$$

The latter expression is independent of $(x, z)$ and goes to zero as $K$ goes to infinity.

LEMMA 4.2. Let $g_{q, 1}, \ldots, g_{q, N(q)}$ be any orthonormal basis for $B_{q}$. Suppose $f \in C(X \times Z)$ and $f$ is analytic on $D_{\sigma}$ as a function of $z_{\sigma}$ for each $\sigma \in \Sigma$. Then

$$
f \in \operatorname{sp}\left(\bigcup\left\{f_{g_{q, j}} \otimes g_{q, j}: 0 \leqq q<\infty, 1 \leqq j \leqq N(q)\right\}\right)^{-} .
$$

Proof. Let $\varepsilon>0$ be given. Select $t, 0 \leqq t<1$, such that $\left\|f-f^{t}\right\|<\varepsilon / 2$. By Lemma 4.1 , select $K$ so large that

$$
\sup \left\{\left|f^{t}(x, z)-\sum_{q=0}^{K} f_{q}^{x}(z) t^{q}\right|:(x, z) \in X \times Z\right\}<\varepsilon / 2 .
$$

Finally, observe that

$$
f_{q}^{x}(z)=\sum_{j=1}^{N(q)} f_{g_{q, j}}(x) g_{q, j}(z)=\sum_{j=1}^{N(q)}\left(f_{g_{q, j}} \otimes g_{q, j}\right)(x, z) .
$$

Now let $X, A, A^{\prime}$ and $\mathfrak{Q}$ be as in the discussion preceding Lemma 3.7, and $\Sigma=\{1, \ldots, r\}$ as above.

LeMma 4.3. $\mathfrak{A}$ consists precisely of those $f \in C(X \times Z)$ which satisfy

(i) $f$ is analytic on $D_{\sigma}$ as a function of $z_{\sigma}$ for each $\sigma \in \Sigma$.

(ii) $f_{g} \in A^{\prime}$ for each $g \in B$.

(iii) $f_{g} \in A$ for each $g \in J=\operatorname{sp}\left(\bigcup\left\{J_{n}: n \geqq 0\right\}\right)^{-}$.

Proof. Suppose $f \in \mathfrak{A}$. (i) and (ii) obviously hold, the latter by Lemma 2.1 and the most elementary facts about integration. In proving (iii), the inclusion $\left\{f_{g}: g \in J\right\} \subset \operatorname{sp}\left(\bigcup\left\{f_{g}: g \in J_{n} \text { for some } n\right\}\right)^{-}$shows that we need only consider $g \in J_{n}$. Using Lemmas 3.8 and 3.12 , we can select sequences $\left\{u_{k}\right\} \subset A \otimes J_{n}$ and $\left\{v_{k}\right\} \subset\left(A^{\prime} \otimes I_{n}\right)+\operatorname{sp}\left(\bigcup\left\{A^{\prime} \otimes B_{q}: 0 \leqq q \neq n\right\}\right)$ such that $\left\|f-f_{k}\right\| \rightarrow 0$ where $f_{k}=$ 
$u_{k}+v_{k}$. Fix $x \in X$. Then $z \rightarrow v_{k}(x, z)$ is in $I_{n}+\operatorname{sp}\left(\bigcup\left\{B_{q}: 0 \leqq q \neq n\right\}\right)$, hence is orthogonal to $g$. Therefore

$$
\begin{aligned}
\left(f_{k}\right)_{g}(x) & =\int_{G} g(z)^{-} f_{k}(x, z) d \lambda(z) \\
& =\int_{G} g(z)^{-} u_{k}(x, z) d \lambda(z)+\int_{G} g(z)^{-} v_{k}(x, z) d \lambda(z)=\left(u_{k}\right)_{g}(x) .
\end{aligned}
$$

Thus $\left(f_{k}\right)_{g}=\left(u_{k}\right)_{g}$. Again, Lemma 2.1 and integration theory yield $\left(u_{k}\right)_{g} \in A$, hence $\left(f_{k}\right)_{g} \in A$. Since $\left\|f_{g}-\left(f_{k}\right)_{g}\right\| \leqq\|g\| \cdot\left\|f-f_{k}\right\| \rightarrow 0$, we get $f_{g} \in A$. Thus (iii) holds.

Conversely, suppose $f \in C(X \times Z)$ satisfies (i)-(iii). Let $g_{q, 1}, \ldots, g_{q, N(q)}$ be an orthonormal basis for $B_{q}$ such that each $g_{q, j}$ is in either $I_{q}$ or $J_{q}$. By (ii) and (iii) we obtain $f_{g_{q, j}} \otimes g_{q, j} \in \mathfrak{A}$ for $0 \leqq q<\infty, 1 \leqq j \leqq N(q)$. By (i) and Lemma $4.2, f$ is in the closed linear span of the $f_{g_{q, j}} \otimes g_{q, j}$. Thus $f \in \mathfrak{A}$.

LEMMA 4.4. If $0 \leqq t<1$ and if $f \in C(X \times Z)$ is analytic on $D_{\sigma}$ as a function of $z_{\sigma}$ for each $\sigma \in \Sigma$ and if $g \in B_{q}$ then $f_{g}(x) t^{2 q}=\int_{G} g(t z)^{-} f(x, t z) d \lambda(z)$ for all $x \in X$.

Proof. Use the observations that $f_{q}^{x}(t z)=t^{q} f_{q}^{x}(z), t^{q} f_{q}^{x}=\left(f_{t}\right)_{q}^{x}, g(t z)=t^{q} g(z)$ and $\int_{G} g(z)^{-} f(x, z) d \lambda(z)=\int_{G} g(z)^{-} f_{q}^{x}(z) d \lambda(z)$.

Proposition 4.5. If $A$ and $A^{\prime}$ are local [resp., holomorphically closed] on $X$ then $\mathfrak{A}$ is local [resp., holomorphically closed] on $X \times Z$ and $M_{\mathfrak{Y}}$.

Proof. In view of our remarks at the beginning of this section, it is enough to prove the result for $X \times Z$. Suppose $f \in C(X \times Z)$ is locally in $\mathfrak{A}$ [resp., 2A-holomorphic] on $X \times Z$. We check that $f$ satisfies the three conditions of Lemma 4.3. (i) is obvious.

(ii) and (iii) reduce to showing that $f_{g}$ is locally in $A^{\prime}$ [resp., $A^{\prime}$-holomorphic] on $X$ for all $g \in B_{q}$, and that $f_{g}$ is locally in $A$ [resp., $A$-holomorphic] on $X$ for all $g \in J_{q}$.

Let $x_{0} \in X$ be given. Let $U$ be an open neighborhood of $x_{0}$ in $X$ and $t$ a positive constant such that $f|V \in \mathfrak{A}| V$ [resp., $f \mid V \in(\mathfrak{A} \mid V)^{-}$] if $V=U \times t D_{1} \times \cdots \times t D_{r}$. Let $f^{\prime} \in \mathfrak{A}$ [resp., $\left.\left\{f_{k}\right\} \subset \mathfrak{A}\right]$ be such that $f\left|V=f^{\prime}\right| V$ [resp., $\left\|\left(f-f_{k}\right) \mid V\right\| \rightarrow 0$ ]. Lemma 4.4 then yields, for $g \in B_{q}, f_{g}\left|U=f_{g}^{\prime}\right| U$ [resp., $\left\|\left(f_{g}-\left(f_{k}\right)_{g}\right)\left|U\|\leqq\| g\|\cdot\|\left(f-f_{k}\right)\right| V\right\| / t^{2 q}$ $\rightarrow 0$ ], from which the desired conclusions can be read.

Now let $X, A$, and $A^{\prime}$ be as in the discussion preceding Theorem 3.16. Trivially, $A^{\prime}$ is holomorphically closed on $X$. In view of Proposition 4.5, to see that the algebra $\mathfrak{A}$ constructed in Theorem 3.17 is local on $X \times Z$ and on $M_{\mathfrak{U}}$ if $J$ is a proper ideal (if $J=B$ then $\mathfrak{A}$ is trivially local, being one-dimensional) it suffices to show that $A$ i; local on $X$. Since $X$ is connected, we need only show that $A$ is analytic on $X$. A variety of routes lead to this conclusion; we choose to quote Wermer's expository text [W1] to point out that $A$ is antisymmetric and is a maximal subalgebra of $C(X)$ [p. 69], hence is analytic on $X$ [p. 62]. Thus we obtain

THEOREM 4.6. The algebra $\mathfrak{A}$ constructed in the proof of Theorem 3.17 is local on $X \times Z$ and on $M_{\mathfrak{r}}$.

Theorem 4.6 has a partial converse, whose proof we leave to the reader. 
Proposition 4.7. With notations as in the beginning of $\S 3$, assume that $\mathfrak{A}$ is local [resp., holomorphically closed] on $X \times Y$. Then

(i) $A$ is local [resp., holomorphically closed] on $X$.

(ii) $B$ is local [resp., holomorphically closed] on $Y$.

(iii) Either $I=\{0\}$ or $A^{\prime}$ is local [resp., holomorphically closed] on $X$.

5. This section will be devoted to miscellany of two types. First, we shall see to what sorts of algebras our construction may lead us. In $\$ 4$ we found conditions guaranteeing that $\mathfrak{A}$ be local; our work on this section will be far more transparent, and will deal for the most part with notions such as essential, pervasive, etc.

Second, we shall be interested in bounded derivations. We shall give examples of such phenomena as nontrivial derivations at one-point parts, and nonlocal derivations in algebras satisfying reasonably strong local belonging conditions. The thread which unites these examples to the main body of our work is the construction, which is used to produce many of the examples.

We begin by defining a number of properties which are frequently studied together. For proofs of some of the unproved assertions below, we refer the reader to [HS].

Let $A$ be a sup-norm algebra on $X . A$ is maximal on $X$ if $A \neq C(X)$, but if whenever $A^{\prime}$ is a sup-norm algebra on $X$ containing $A$, either $A^{\prime}=A$ or $A^{\prime}=C(X)$; automatically $S_{A}=X . A$ is essential if there is no proper closed subset $F$ of $X$ such that $A$ contains every function in $C(X)$ vanishing identicall $y^{\prime}$ on $F$; this property is independent of the choice of boundary $X$ for $A$. $A$ is pervasive on $X$ if whenever $F$ is a proper closed subset of $X, A \mid F$ is dense in $C(F)$; again, this implies $S_{A}=X . A$ is analytic on $X$ if no function of $A$ other than the zero function vanishes identically on a nonvoid open subset of $X . A$ is antisymmetric if it contains no nonconstant real-valued functions; this property is independent of the choice of boundary $X$ for $A$. The following chain of implications is well known: $A$ is maximal and essential on $X \Rightarrow A$ is a proper pervasive subalgebra of $C(X) \Rightarrow A$ is analytic on $X \Rightarrow A$ is an integral domain $\Rightarrow A$ is antisymmetric $\Rightarrow A$ is essential.

We shall present criteria for $\mathfrak{A}$ to be maximal, essential, etc. Observe that some of the properties, e.g., being essential, occur virtually all the time while others, e.g., being maximal, occur quite rarely. Proofs are easy, and are left to the reader.

Criterion 5.1. With notations as in the discussion preceding Lemma 3.7, except that $I$ need not be proper, $\mathfrak{A}$ will be maximal (on $S_{\mathfrak{l}}$, of course) precisely when $X$ consists of a single point and $\Sigma$ consists of just one element.

Criterion 5.2. With notations as in the beginning of $\S 3$, $\mathfrak{A}$ will be essential if at least one of the following three conditions holds:

(i) $A^{\prime}$ is essential.

(ii) $A$ is essential and $I=\{0\}$.

(iii) $B$ is essential.

Criterion 5.3. With notations as in the beginning of $\S 3, \mathfrak{A}$ will be pervasive (on $S_{\mathfrak{U}}$, of course) precisely when at least one of the following five conditions holds: 
(i) $X$ consists of a single point and $B$ is pervasive.

(ii) $Y$ consists of a single point and $A$ is pervasive.

(iii) $Y$ consists of a single point, $I=B$, and $A^{\prime}$ is pervasive.

(iv) $A=C(X)$ and $B=C(Y)$.

(v) $A^{\prime}=C(X)$ and $I=B=C(Y)$.

Criterion 5.4. With notations as in the beginning of $\S 3, \mathfrak{A}$ will be analytic on $S_{A^{\prime}} \times S_{B}$ precisely when either

(i) $A$ is analytic on $S_{A^{\prime}}, B$ is analytic on $S_{B}$, and $I=\{0\}$.

(ii) $A^{\prime}$ is analytic on $S_{A^{\prime}}$ and $B$ is analytic on $S_{B}$.

If any of these conditions holds then $\mathfrak{A}$ is analytic on $S_{\mathfrak{A}}$.

Similarly, if $\mathfrak{A}$ is analytic on $M_{\mathfrak{A}}$ then at least one of the following three conditions holds:

(a) $A$ is analytic on $M_{A}, B$ is analytic on $Y$, and $I=\{0\}$.

(b) $A$ is analytic on $M_{A}, B$ is analytic on $Y, A^{\prime}$ is analytic on $X$, and $\{0\} \neq I \neq B$.

(c) $A^{\prime}$ is analytic on $X, B$ is analytic on $Y$, and $I=B$.

Conversely, (a) or (c) implies that $\mathfrak{A}$ is analytic on $M_{\mathfrak{r}}$.

Criterion 5.5. With notations as in the beginning of $\S 3$, if $\mathfrak{A}$ is an integral domain then either $A^{\prime}$ and $B$ are integral domains, or $A$ and $B$ are integral domains and $I=\{0\}$.

Criterion 5.6. With notations as in the beginning of $\S 3, \mathfrak{A}$ is antisymmetric precisely when either $A$ and $B$ are antisymmetric and $I \neq B$, or $A^{\prime}$ and $B$ are antisymmetric.

Combining these criteria, we obtain the

Proposition 5.7. The algebra $\mathfrak{A}$ constructed in the proof of Theorem 3.16 [resp., Theorem 3.17] for the case $I \neq B$ [resp., $J \neq R]$ is always essential and antisymmetric, and is never maximal or pervasive. It is analytic on $X \times G$ precisely when it is analytic on $M_{\mathfrak{A}}$; this happens precisely when $I=\{0\}[$ resp., $J=\{0\}$ ], which is precisely when $\mathfrak{A}$ is an integral domain.

The parts in $M_{\mathfrak{A}}$ may be of some interest, especially in view of recent work by Garnett [Ga]. We can obtain a wide variety of parts (including disconnected ones) via our construction. Recall that $\varphi \sim \varphi^{\prime} \Leftrightarrow\left\|\varphi-\varphi^{\prime}\right\|_{A^{*}}<2$ is an equivalence relation on $M_{A}[\mathrm{Gl}]$, and that the equivalence classes are the (Gleason) parts for $A$.

Proposition 5.8. With notations as in the beginning of $\S 3$, suppose the $A$ and $A^{\prime}$ parts relations coincide on $X$. Then the parts for $\mathfrak{A}$ are precisely the sets $P=$ $\left(P_{1} \times P_{2}\right) \cap M_{\mathfrak{V}}$ where $P_{1}$ is a part for $A$ in $M_{A}$ and $P_{2}$ is a part for $B$ in $Y$.

COROLLARY 5.9. The set $\left\{\varphi_{0}\right\} \times$ (hull $\left.(J) \cap \prod_{\gamma=1}^{r} D_{\sigma}\right)$ is a single part for the algebra constructed in the proof of Theorem 3.17 for the case $J \neq R$.

Proposition 5.10. With notations as in the beginning of $\S 3$, let $N$ denote any cardinal number and assume $\mathfrak{A}$ has a system of $N$ generators. Then $B$ has a system of $N$ or fewer generators. If $I \neq B$ then $A$ has a system of $N$ or fewer generators, and if $I \neq\{0\}$ then $A^{\prime}$ has a system of $N$ or fewer generators. 
Conversely, let $N_{1}, N_{2}, N_{3}, N_{4}$ denote cardinal numbers. Assume $A$ has a system of $N_{1}$ generators, $B$ has a system of $N_{2}$ generators, $A^{\prime}$ is the closed $A$ module spanned by 1 and $N_{3}$ other functions, and $I$ is the closed ideal of $B$ generated by $N_{4}$ elements of I. Then $\mathfrak{A}$ has a system of $N_{1}+N_{2}+N_{3} N_{4}$ or fewer generators.

Proof. The first half is trivial. For the second, let $\left\{a_{n_{1}}: n_{1} \in N_{1}\right\},\left\{b_{n_{2}}: n_{2} \in N_{2}\right\}$, $\left\{a_{n_{3}}^{\prime}: n_{3} \in N_{3}\right\}$ and $\left\{i_{n_{4}}: n_{4} \in N_{4}\right\}$ be the four systems of generators described in the hypotheses. It is easy to see that the union of the systems $\left\{a_{n_{1}} \otimes 1: n_{1} \in N_{1}\right\}$, $\left\{1 \otimes b_{n_{2}}: n_{2} \in N_{2}\right\}$, and $\left\{a_{n_{3}}^{\prime} \otimes i_{n_{4}}: n_{3} \in N_{3}, n_{4} \in N_{4}\right\}$ generates $\mathfrak{A}$.

COROLlARY 5.11. If $A$ and $B$ are finitely generated, if $A^{\prime}$ is a finitely generated closed $A$-module, and if $I$ is a finitely generated closed ideal in $B$, then $\mathfrak{A}$ is finitely generated.

We are now almost ready to begin the examples. We need one more fact which will make Proposition 5.10 useful.

LEMMA 5.12. Let $r$ be a positive integer, let $\bar{D}^{r}$ have coordinates $z=\left(z_{1}, \ldots, z_{r}\right)$, let $A^{\prime}=C\left(T^{r}\right)$, and let $A$ be the restriction to $T^{r}$ of the functions continuous on $\bar{D}^{r}$ and analytic on $D^{r}$. Then there exists $g \in A^{\prime}$ such that $A^{\prime}$ is the closed A-module generated by 1 and $g$.

Proof. First, suppose $r=1$ and $z=z_{1}$. Define $h$ on $T$ by $h(z)=\sum_{n=1}^{\infty} \bar{z}^{2^{N}} / n$ ! where the bar denotes complex conjugation. Consideration of the functions $m ! z^{2^{m}-2^{N}} h(z)$ for $m>N$ shows that $\bar{z}^{2^{N}}$ is in the closed $A$-module generated by 1 and $h$, for every positive integer $N$.

Next let $r$ be arbitrary and define $g$ by $g\left(z_{1}, \ldots, z_{r}\right)=h\left(z_{1}, \ldots, z_{r}\right)$ where $h$ is as above. Then our discussion of $h$ shows that $\left(z_{1}, \ldots, z_{r}\right)^{-2^{N}}$ is in the closed $A$ module generated by $g$, for every positive integer $N$. It is then easy to complete the proof with the aid of the Stone-Weierstrass theorem.

Let $A$ be a sup-norm algebra, $L$ a linear functional on $A, \varphi \in M_{A} . L$ is called a derivation of $A$ at $\varphi$ if $L$ satisfies the usual product rule for derivatives: $L(f g)$ $=L(f) \cdot \varphi(g)+\varphi(f) \cdot L(g)$. This is equivalent to annihilating both the constants and the ideal $A_{\varphi}^{2}$. This suggests considering also functionals which annihilate $A_{\varphi}^{n}$ for some $n$; if $L$ is also continuous, we write $L \in\left(A_{\varphi}^{n}\right)^{\perp}$ where $\left(A_{\varphi}^{n}\right)^{\perp} \subset A^{*}$.

The most familiar examples come from differentiation of analytic functions. These derivations are trivially local in the following sense: a linear functional $L$ on $A$ is local at $\varphi \in M_{A}$ if whenever $f \in A$ vanishes on a neighborhood of $\varphi$ in $M_{A}$, it follows that $L(f)=0$. Since analytic functions form one of the models for supnorm algebra theory, the next obvious question is, when can we guarantee that a derivation be local?

It has been known at least since the appearance of Eva Kallin's famous example [K] that continuous nonlocal derivations may exist. On the other hand, the results of [S1] show that the hypothesis of unique representing measures precludes the possibility of continuous nonlocal derivations. 
It is suggestive that the first nonlocal derivation occurs in the first nonlocal algebra, and an obvious conjecture arises. The conjecture is rendered plausible by the fact that both uses of the term "local" stipulate that all the information one wants can be obtained by looking at small open sets. Nonetheless, as we shall see in Examples 5.13 through 5.15 below, the conjecture is false even under very strong hypotheses.

EXAMPLE 5.13. Let $n$ be an integer greater than $1 . A$ and $A^{\prime}$ will be as in the discussion preceding Theorem 3.16, i.e., $A^{\prime}=C\left(T^{2}\right)$ and $A$ is a Dirichlet algebra on $X=T^{2}$ having a one-point part $\varphi_{0}$ off $X=S_{A} . B$ will be the disc algebra on $Z=\bar{D}$ (with coordinate $z$ ) and $I$ will be the closed ideal in $B$ generated by $z^{n}$ (that is, $I$ consists of those functions in $B$ which vanish at 0 along with their first $n-1$ derivatives). $\mathfrak{A}$ is just the algebra constructed in proving Theorem 3.17 for the case $r=1, J$ the ideal of $C\left[X_{1}\right]$ generated by $X_{1}^{n}$. Therefore $\left(\mathfrak{A}_{\varphi}^{q}\right)^{-} /\left(\mathfrak{A}_{\varphi}^{q+1}\right)^{-}$is onedimensional if $0 \leqq q<n$, and $\left(\mathfrak{H}_{\varphi}^{q}\right)^{-}=\left(\mathfrak{A}_{\varphi}^{q+1}\right)^{-}$if $q \geqq n$. By Theorem 4.6, $\mathfrak{A}$ is local (on $M_{\mathfrak{U}}$ ). By Criterion $5.6 \mathfrak{A}$ is antisymmetric, hence essential.

Since hull $(J)=\{0\}$, Corollary 5.9 implies that $\varphi$ is a one-point part for $\mathfrak{A}$. Since there is a nonzero bounded derivation of $\mathfrak{A}$ at $\varphi$ because $\mathfrak{A}_{\varphi} \neq\left(\mathfrak{A}_{\varphi}^{2}\right)^{-}$, we see that just about the weakest imaginable converse to a recent result of Andrew Browder is false. Browder shows [B] that if $\varphi$ is not isolated in $M_{A}$ in the $A^{*}$ norm topology, then $\varphi$ supports a (not necessarily continuous) nontrivial derivation of $A$.

The function $1 \otimes z$ is in $\mathfrak{A}$ and vanishes on the neighborhood $M_{A} \times\{0\}$ of $\varphi$ in $M_{\mathfrak{A}}$, but Lemma 3.12 implies it is not in $\left(\mathfrak{A}_{\varphi}^{2}\right)^{-}$; hence there is a nonlocal bounded derivation of $\mathfrak{A}$ at $\varphi$.

The function $1 \otimes z^{k}$ provides a generator for $\left(\mathfrak{A}_{\varphi}^{k}\right)^{-} /\left(\mathfrak{A}_{\varphi}^{k+1}\right)^{-}$for $1 \leqq k \leqq n-1$.

ExAmple 5.14. Let $X=T, A^{\prime}=C(X), A$ the restriction to $X$ of the disc algebra. Let $B$ be the disc algebra on $Z=\bar{D}$, let $I$ be the closed ideal of $B$ generated by $z^{2}$, let $\varphi_{0}=0 \in \bar{D}=M_{A}$, and $\varphi=\left(\varphi_{0}, 0\right) \in M_{\mathfrak{U}}$ where $\mathfrak{A}$ is constructed in the usual way. By Proposition 4.5, $\mathfrak{A}$ is local (on $M_{\mathfrak{A}}$ ). Criterion 5.6 says $\mathfrak{A}$ is antisymmetric. Proposition 5.10 and Lemma 5.12 show that $\mathfrak{A}$ is generated by only three functions. By Lemma $3.12\left(\mathfrak{A}_{\varphi}^{n}\right)^{-} \neq\left(\mathfrak{\Re}_{\varphi}^{n+1}\right)^{-}$for all $n$.

The function $1 \otimes z$ is in $\mathfrak{A}$ and vanishes on the neighborhood $M_{A} \times\{0\}$ of $\varphi$ in $M_{\mathfrak{U}}$, but Lemma 3.12 implies $1 \otimes z \notin\left(\mathfrak{A}_{\varphi}^{2}\right)^{-}$. Thus $\varphi$ supports a nonlocal continuous derivation of $\mathfrak{A}$.

This example was called to the author's attention by Professor A. M. Gleason, and it led the author to the construction in $\$ 3$.

Note that if we let $I$ be generated by $z$ rather than $z^{2}$, the resulting algebra $\mathfrak{A}$ is a familiar Dirichlet algebra on $T^{2}$ [W1, p. 69], and the same argument shows that it is triply generated, a fact which may be of independent interest.

EXAMPLE 5.15. Let $m>1$ be an integer, let $X=$ bdry $_{C^{m}}\left(D^{m}\right)$, let $A^{\prime}=C(X)$ and let $A$ be the polydisc algebra on $\bar{D}^{m}$ restricted to $X$, so $S_{A}=T^{m}$ and $M_{A}=\bar{D}^{m}$. It is not difficult to show that $A$ is holomorphically closed on $X$.

Let $B$ be the disc algebra on $Z=\bar{D}$, let $I$ be the closed ideal of $B$ generated by $z^{2}$, 
let $\varphi_{0}=0 \in \bar{D}^{m}=M_{A}$, and let $\varphi=\left(\varphi_{0}, 0\right) \in M_{\mathfrak{U}}$ where $\mathfrak{A}$ is constructed in the usual way. By Proposition 4.5, $\mathfrak{A}$ is holomorphically closed (on $M_{\mathfrak{U}}$ ). Criterion 5.6 says $\mathfrak{A}$ is antisymmetric. From Lemma 3.12 we see that $\left(\mathfrak{Q}_{\varphi}^{n}\right)^{-} \neq\left(\mathfrak{Q}_{\varphi}^{n+1}\right)^{-}$for all $n$.

As usual, the function $1 \otimes z$ is in $\mathfrak{A}$ and vanishes on the neighborhood $M_{A} \times\{0\}$ of $\varphi$ in $M_{\mathfrak{A}}$, but $1 \otimes z \notin\left(\mathfrak{A}_{\varphi}^{2}\right)^{-}$, so a nonlocal continuous derivation of $\mathfrak{A}$ at $\varphi$ exists. Thus even holomorphic closure of $\mathfrak{U}$-as strong a local belonging property as one could wish-need not guarantee localness of derivations.

EXAMPLE 5.16. Let $X, A, A^{\prime}$ and $\varphi_{0}$ be as in Example 5.13. Let $Z=\prod_{n=1}^{\infty} \bar{D}_{n}$ where $\bar{D}_{n}$ is a closed disc with coordinate $z_{n}$, let $B=P(Z)$, and let $I$ be the closed ideal of $B$ generated by the functions $\left\{z_{n}^{n}: n=1,2, \ldots\right\}$. Then hull $(I)=\{0\}$ and $I$ is a homogeneous ideal in $B$ (in our nonalgebraic sense of the word "homogeneous"). Construct $\mathfrak{A}$ as usual, so (Criterion 5.6) $\mathfrak{A}$ is antisymmetric, and let $\varphi=\left(\varphi_{0}, 0\right) \in M_{\mathfrak{A}}$, so (Proposition 5.8) $\varphi$ is a one-point part for $\mathfrak{A}$.

Corollary 3.10 can be used to see that $1 \otimes z_{m+1}^{n} \notin\left(\mathfrak{A}_{\varphi}^{n+1}\right)^{-}$while of course $1 \otimes z_{n+1}^{n} \in \mathfrak{A}_{\varphi}^{n}$. Thus $\left(\mathfrak{A}_{\varphi}^{n}\right)^{-} \neq\left(\mathfrak{A}_{\varphi}^{n+1}\right)^{-}$for all $n$, although $\varphi$ is a one-point part. Note also that $1 \otimes z_{2}$ vanishes on the neighborhood $M_{A} \times\{0\}$ of $\varphi$ in $M_{\mathfrak{U}}$ but $1 \otimes z_{2} \notin\left(\mathfrak{H}_{\varphi}^{2}\right)^{-}$, hence there is a nonlocal continuous derivation of $\mathfrak{A}$ at $\varphi$.

Close inspection will reveal that the nonlocal derivations we have been producing are local somewhere on $M_{\mathfrak{N}}$, though not at the point $\varphi$ under consideration. For example, the nonlocal derivation indicated in Example 5.13 is actually local at every point of $X \times Z$. We now indicate how to construct nowhere local derivations.

Example 5.17. For $j=1,2$ let $A_{j}$ be a sup-norm algebra on $X_{j}=M_{A_{j}}$ and let $L_{j}$ be a continuous nonlocal derivation of $\varphi_{j} \in X_{j}$. Let $X$ denote the union of $X_{1}$ and $X_{2}$, disjoint except for the identification of $\varphi_{1}$ with $\varphi_{2}$; let $\varphi$ denote this common point of $X$. Let $A=\left\{f \in C(X): f \mid X_{j} \in A_{j}\right.$ for $\left.j=1,2\right\}$. Then $M_{A}=X$.

Define $L$ by $L(f)=L_{1}\left(f \mid X_{1}\right)+L_{2}\left(f \mid X_{2}\right)$. $L$ is then a bounded derivation of $A$ at $\varphi$ which is local at no point of $X$.

Many properties of interest which $A_{1}$ and $A_{2}$ may have carry over to $A$, e.g., localness, holomorphic closure, antisymmetry, essentiality. If $\varphi_{1}$ and $\varphi_{2}$ are onepoint parts, so is $\varphi$. Thus combining our previous examples, one can construct nowhere local continuous point derivations in algebras satisfying all sorts of decent conditions.

All of our examples so far have utilized analytic structures in the maximal ideal space, even if the analytic structure has not been at the point in question. We close by using a "Swiss cheese" algebra to point out that even with no analytic structure anywhere in $M_{A}$, it is possible to have $\left(A_{\varphi}^{n}\right)^{-} \neq\left(A_{\varphi}^{n+1}\right)^{-}$for all $n$.

EXAMPLE 5.18. Let $\left\{D_{k}: k=1,2, \ldots\right\}$ be a sequence of mutually disjoint discs (with centers $b_{k}$ and radii $r_{k}$ ) contained in $D_{0}$, the open unit disc in $\boldsymbol{C}$. Assume $\sum_{k=1}^{\infty} r_{k}<\infty$ and let $X=\bar{D}_{0}-\bigcup_{k=1}^{\infty} D_{k}$, a compact set in the plane. Let $A$ denote the uniform closure on $X$ of the rational functions whose poles lie off $X . A$ is a sup-norm algebra on $X$, and $M_{A}=X$ (see [W1, p. 77]). 
It is possible to choose the $D_{k}$ so that $\bigcup_{k=1}^{\infty} D_{k}$ is dense in $D_{0}$ and does not contain 0 , and so that $\sum_{k=1}^{\infty} r_{k} /\left(\left|b_{k}\right|-r_{k}\right)^{m}<\infty$ for $m=0,1,2, \ldots$ Then $X$ has no planar interior, so $M_{A}$ has no analytic structure of any kind. Let $\varphi \in M_{A}$ be evaluation at 0 .

Let $T_{k}$ be the circle bounding $D_{k}$. $\mu$ will be the complex Borel measure on $X$ supported by $\bigcup_{k=0}^{\infty} T_{k}$ and given by $d \mu(z)=d z / 2 \pi i z$ on $T_{0}$ and $d \mu(z)=-d z / 2 \pi i z$ on $T_{k}, k>0$. For $m=0,1,2, \ldots$ let $d \mu_{m}(z)=z^{-m} d \mu(z) . \mu_{m}$ is a complex measure; in fact,

$$
\left\|\mu_{m}\right\| \leqq 1+\sum_{k=1}^{\infty} r_{k} /\left(\left|b_{k}\right|-r_{k}\right)^{m+1}<\infty
$$

It is easy to show that $\mu_{m}$ annihilates $A_{\varphi}^{m+1}$. However, clearly $z^{m} \in A_{\varphi}^{m}$ but $\int z^{m} d \mu_{m}(z)=\int d \mu(z)=1$. Thus $\left(A_{\varphi}^{m}\right)^{-} \neq\left(A_{\varphi}^{m+1}\right)^{-}$for all $m$.

Wermer [W2] has apparently proved two interesting facts along these lines. First, if $\sum_{k=1}^{\infty} r_{k} \cdot\left|\log r_{k}\right|<\infty$ then there exists a nontrivial bounded derivation at almost every point of $X$. Second, and of great interest, is the fact that the $D_{k}$ may be chosen in such a manner that there exists no bounded derivation of $A$ at any point of $X$.

\title{
REFERENCES
}

[B] A. Browder, Point derivations in function algebras, J. Functional Analysis 1 (1967), 22-27.

[Ga] J. Garnett, A topological characterization of Gleason parts, Pacific J. Math. 20 (1967), 59-63.

[Ge] B. R. Gelbaum, Tensor products and related questions, Trans. Amer. Math. Soc. 103 (1962), 525-548.

[GI] A. M. Gleason, Function algebras, Seminars on analytic functions, Institute for Advanced Study, Princeton, N. J., 1957.

[GR] R. C. Gunning and H. Rossi, Analytic functions of several complex variables, PrenticeHall, Englewood Cliffs, N. J., 1965.

[HS] K. Hoffman and I. M. Singer, Maximal algebras of continuous functions, Acta Math. 103 (1960), 217-241.

[K] E. Kallin, A nonlocal function algebra, Proc. Nat. Acad. Sci. U.S.A. 49 (1963), 821-824.

[R] C. E. Rickart, Analytic phenomena in general function algebras. I, Pacific J. Math. 18 (1966), 361-377.

[S1] S. J. Sidney, Point derivations in certain sup-norm algebras, Trans. Amer. Math. Soc. 131 (1968), 119-127.

[S2] — , Powers of maximal ideals in function algebras, Thesis, Harvard Univ., Cambridge, Mass., 1966.

[W1] J. Wermer, Banach algebras and analytic functions, Advances in Math. 1 (1961), 51-102.

[W2] - Bounded point derivations on certain Banach algebras, J. Functional Analysis 1 (1967), 28-36.

[ZS] O. Zariski and P. Samuel, Commutative algebra. II, Van Nostrand, Princeton, N. J., 1960.

\author{
HARVARD UNIVERSITY, \\ Cambridge, Massachusetts \\ YALE UNIVERSITY, \\ New Haven, Connecticut
}

